University of Louisville

ThinkIR: The University of Louisville's Institutional Repository

Electronic Theses and Dissertations

8-2013

\title{
Evaluation of a children's hospital's direct admission process to improve outcomes and efficiency of care.
}

Scott R. Hoover

University of Louisville

Follow this and additional works at: https://ir.library.louisville.edu/etd

\section{Recommended Citation}

Hoover, Scott R., "Evaluation of a children's hospital's direct admission process to improve outcomes and efficiency of care." (2013). Electronic Theses and Dissertations. Paper 632.

https://doi.org/10.18297/etd/632

This Doctoral Dissertation is brought to you for free and open access by ThinkIR: The University of Louisville's Institutional Repository. It has been accepted for inclusion in Electronic Theses and Dissertations by an authorized administrator of ThinkIR: The University of Louisville's Institutional Repository. This title appears here courtesy of the author, who has retained all other copyrights. For more information, please contact thinkir@louisville.edu. 
EVALUATION OF A CHILDREN'S HOSPITAL'S DIRECT ADMISSION PROCESS TO IMPROVE OUTCOMES AND EFFICIENCY OF CARE

\author{
By \\ Scott R. Hoover \\ B.S. Mathematics, University of Louisville, 2009 \\ M.S. Industrial Engineering, University of Louisville, 2010
}

\author{
A Dissertation \\ Submitted to the Faculty of the \\ University of Louisville \\ J.B. Speed School of Engineering \\ in Partial Fulfillment of the Requirements \\ for the Professional Degree
}

Doctor of Philosophy

Department of Industrial Engineering
University of Louisville
Louisville, Kentucky

August 2013 



\section{EVALUATION OF A CHILDREN'S HOSPITAL'S DIRECT ADMISSION PROCESS TO IMPROVE OUTCOMES AND EFFICIENCY OF CARE}

\section{By}

Scott R. Hoover

B.S. Mathematics, University of Louisville, 2009

M.S. Industrial Engineering, University of Louisville, 2010

\section{A Dissertation Approved on}

July 15, 2013

By the following Dissertation Committee Members:

Dr. Grady T. Holman, I.E. Department, Co-Chair

Dr. Gail W. DePuy, I.E. Department, Co-Chair

Dr. Charles Timothy Hardin, I.E. Department

Dr. Vicki Montgomery, Department of Pediatrics 


\section{ACKNOWLEDGEMENTS}

I would first like to thank my dissertation directors Dr. Talley Holman and Dr. Gail DePuy for all of their guidance and support. I would like to offer a special thanks to Dr. Holman for taking me under his wing, teaching me so much about performing proper research, and providing me with many opportunities that I would not have had otherwise. I would also like to offer a special thanks to Dr. DePuy for encouraging me to pursue a career as an Industrial Engineer. I would also like to thank Dr. Tim Hardin, whose knowledge and kind heart have been a great help over the last few years. In addition, I would like to thank Dr. Vicki Montgomery for allowing me the opportunity to perform my dissertation work at Kosair Children's Hospital.

I would also like to thank my parents Todd and Karen, whose love, support, and guidance have helped me throughout all of my schooling. They are the best parents a person could ever have.

Next, I would like to thank all of my friends. Throughout all of this, I have come to realize the importance of having good friends to support and be there for you.

Most of all, I would like to thank my wife Sarah, whose unconditional love and encouragement have helped me get through these last four years of my life. I would not have been able to do it without her. 


\title{
ABSTRACT \\ EVALUATION OF A CHILDREN'S HOSPITAL'S DIRECT ADMISSION PROCESS TO IMPROVE OUTCOMES AND EFFICIENCY OF CARE
}

\author{
Scott R. Hoover
}

July 15,2013

Delays in healthcare process pose problems in hospitals today (Lane, Monefeldt, \& Rosenhead, 2000; Horwitz \& Bradley, 2009; Stone, Boehme, Mundorff, Maloney, \& Sriastava, 2010). These delays create risk for patient by preventing timely delivery of care. The purpose of this research was to evaluate the Admission Express Unit (AEU) of Kosair Children's Hospital $(\mathrm{KCH})$ for potential areas for improvement to aide in decreasing the amount of time required for patients to be processed through the unit. This research was a prospective study using direct observations from both the patient's point of view and the nurse's point of view. Also, one-on-one interviews were performed with doctors, nurses, nurse managers, and administrators who work in or with the AEU to gain a complete understanding of the process.

From this research, several observations were made about the AEU that identified some of the issues related to increased amount of time for patients to travel through the system. First, it was discovered that $37 \%$ of the deviated events or failures, which resulted in delays, were found in the Nurse Assessment step. Secondly, it was discovered that the Intake Process had the highest average time to recover from delays, averaging 
32 minutes. Thirdly, it was discovered that, according to the staff and personnel of the AEU, there are five problematic process steps, out of approximately thirty, in the AEU process: 1) Patient Pre-Arrival Work, 2) Patient Roomed AEU, 3) Physician Arrives, 4) Make/View/Receive Orders, and 5) Call Report to Floor. Next, it was discovered that of the five problems identified by the staff and personnel, three of the problem areas are dependent on one another; Patient Pre-Arrival Work, Physicians Arrives, and Make/View/Receive Orders. Finally, it was discovered that the Call-in/Patient PreArrival Process is the most important process of the AEU relative to the amount of time patients spend in the unit because it is the first step the patient incurs. If it does not go smoothly the remaining processes are affected. Also, the remaining process steps cannot be executed until the Call-in/Patient Pre-Arrival Process is successfully completed.

From these findings, a list of recommendations was created to provide to Kosair Children's Hospital that could potentially assist in improving the AEU process. First, the AEU needs to identify one point of contact for the primary care physicians to call to admit patients. Also, whoever the contact person is, they need to develop an intake form that, when completed, contains the necessary patient information while identifying the acuity of the patient. Secondly, it is recommended that $\mathrm{KCH}$ train its new residents each year in the policies and procedures of the AEU so that each resident is aware of such things as the ability to decline and refer a patient to the emergency department based on acuity and the 15 minute time limit to see each patient upon arrival to the AEU. Thirdly, the AEU needs to reevaluate their policies and agree that consultations and most treatments will be performed in the patient's room in the general medical and surgical care areas and not in the AEU. Finally, it is recommended that the AEU not be used as 
an area for teaching new residents as the AEU is an express unit and the teaching of the residents only delays the process of getting patients in and out. 


\section{TABLE OF CONTENTS}

ACKNOWLEDGEMENTS ...........................................................................ii

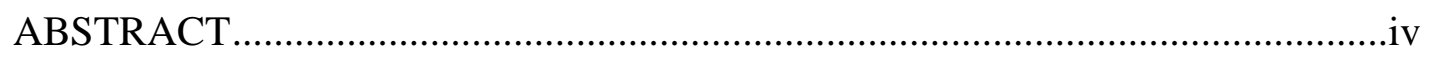

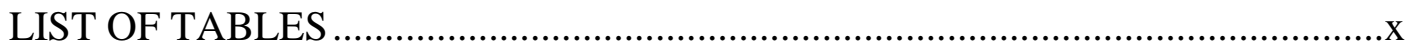

LIST OF FIGURES .............................................................................

CHAPTER 1: INTRODUCTION ...............................................................

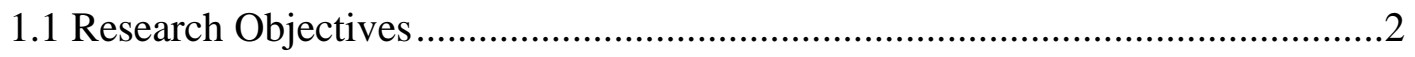

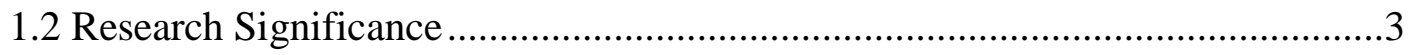

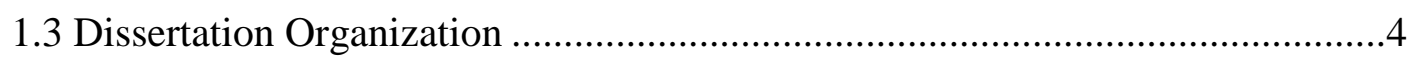

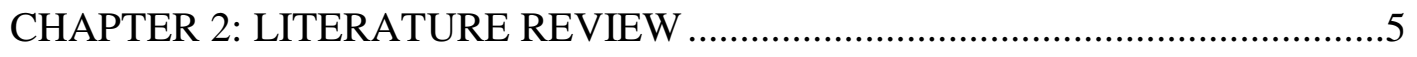

2.1 Systems Engineering Initiative for Patient Safety .....................................6

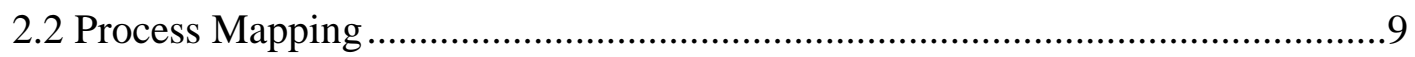

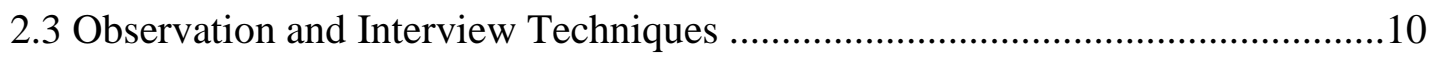

CHAPTER 3: WORKFLOW FAILURES AND RECOVERIES IN AN ADMISSIONS UNIT ............................................................ 12

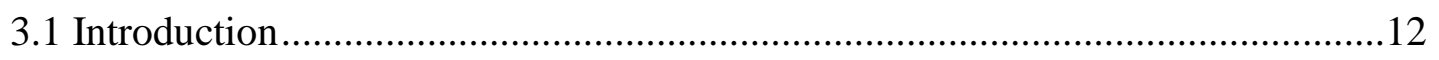

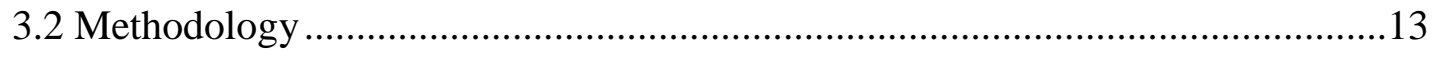

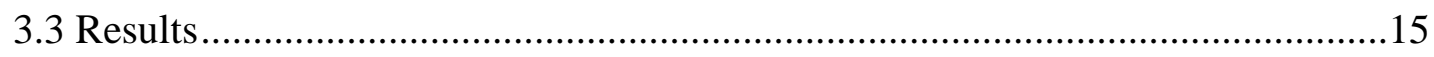

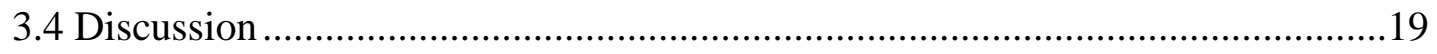


3.5 Conclusion . 22

3.5.1 Future Work. 22

CHAPTER 4: HOW AN EMPLOYEE'S POSITION AFFECTS THEIR PERCEPTION OF THE WORKFLOW ......................................24

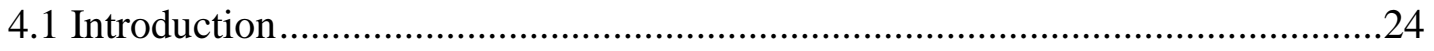

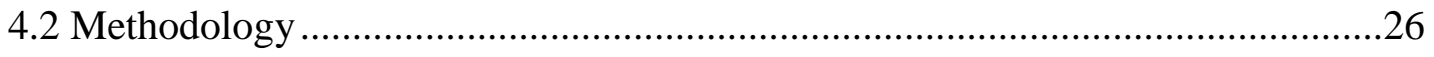

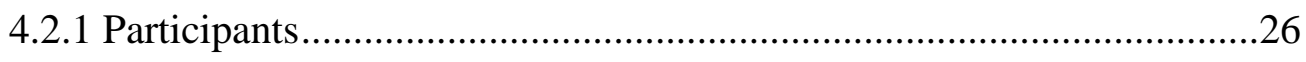

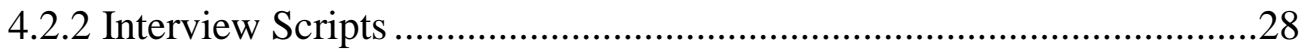

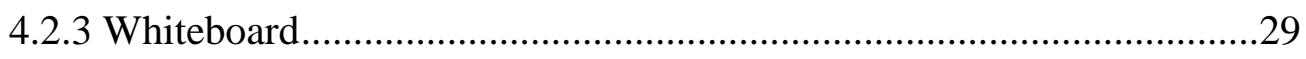

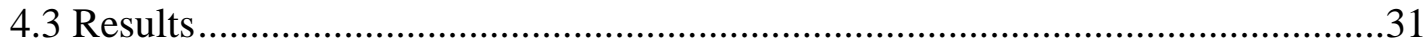

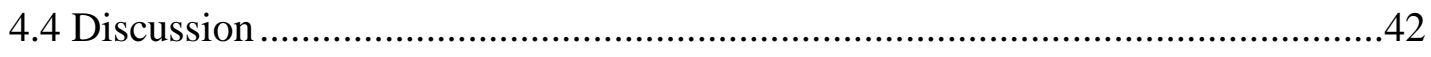

4.4.1 Workflow Detail .....................................................................42

4.4.2 Importance of Delay, Inefficiencies, and Personnel to a Group ...........44

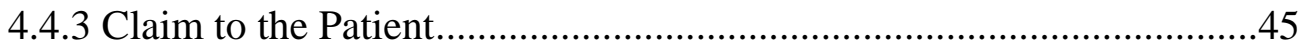

4.4.4 Potential Limitations ................................................................46

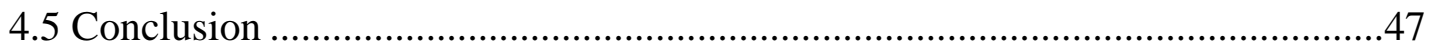

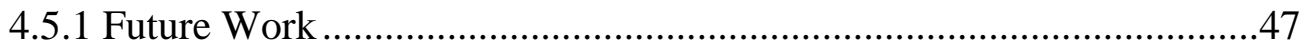

CHAPTER 5: ASSESSMENT OF A HOSPITAL RAPID ADMISSIONS UNIT

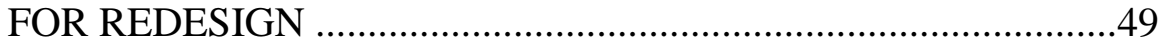

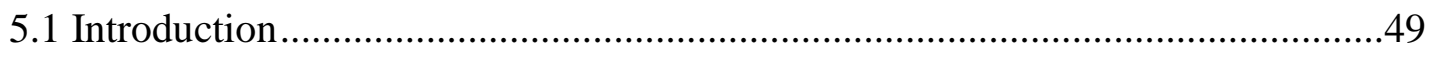

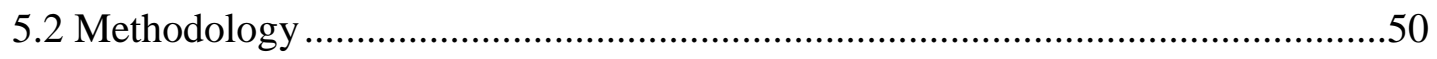

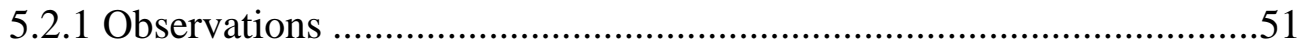


5.2.2 Interviews

5.2.3 Document Collection 52

5.3 Results .53

5.4 Discussion .58

5.4.1 Potential Limitations .65

5.5 Conclusion .66

5.5.1 Future Work 67

CHAPTER 6: CONCLUSIONS AND FUTURE RESEARCH. .68

6.1 Future Work .69

REFERENCES .71

APPENDIX A: DEVIATED EVENTS OR FAILURES OF THE AEU .77

APPENDIX B: ANOVA.. .80

APPENDIX C: INTERVIEW SCRIPT FOR NURSE MANAGERS, NURSES, AND UNIT SECRETARY .81

APPENDIX D: INTERVIEW SCRIPT FOR DOCTOR INTERVIEW .86 APPENDIX E: INTERVIEW SCRIPT FOR ADMINISTRATOR INTERVIEW ..91

CURRICULUM VITAE. .96 


\section{LIST OF TABLES}

Table 1: Examples of Deviated Events or Failures............................................17

Table 2: Summary Table of Deviated Events and/or Failures of the AEU ..............18

Table 3: Abbreviation Meanings of Key for Workflows........................................ 31

Table 4: Summary Table of Doctor's Delays and Inefficiencies............................33

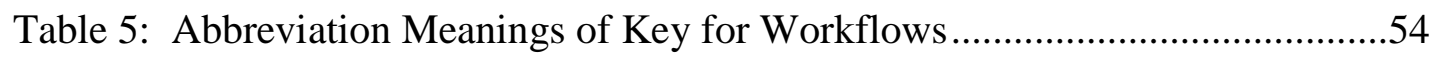

Table 6: Failures in the AEU and Potential Fixes.................................................59

Table 7: Deviated Events and/or Failures of the AEU ...........................................77 


\section{LIST OF FIGURES}

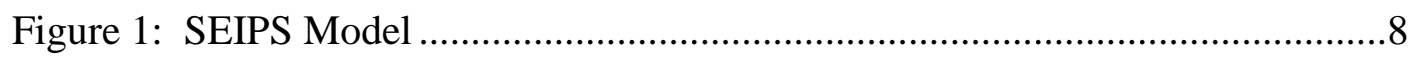

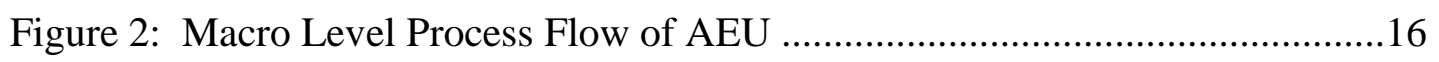

Figure 3: Percentage of Deviated Events and/or Failures in AEU Process Steps ....18

Figure 4: Magnetic Whiteboard and AEU Process Steps ......................................30

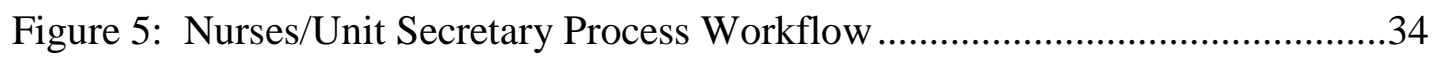

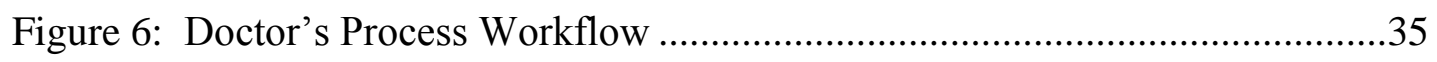

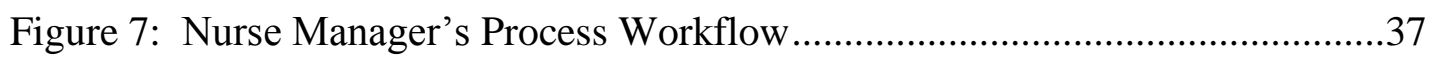

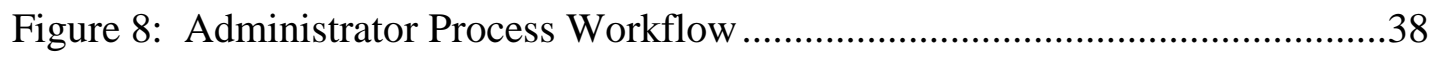

Figure 9: Combined AEU Process Workflow .................................................41

Figure 10: Representation of Detailed Provided..............................................43

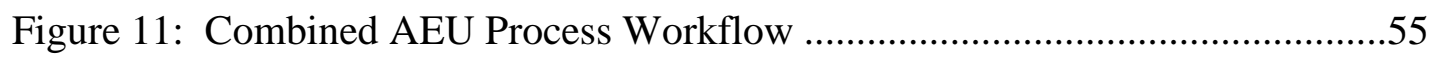

Figure 12: Analysis of Variance of the Macro Level Process Steps.........................80 


\section{CHAPTER 1 INTRODUCTION}

The admission process is the first process most patients go through when being admitted to the hospital. The process normally consists of gathering patient information and medical history, placing a personal identification bracelet on the patient, and having the patient or guardian sign consent forms for the hospital so treatment may be performed. While this is the norm for most hospital admission processes, variations exist between hospitals relative to the how these tasks are performed and in what order. Such is the case with the Admission Express Unit (AEU) of Kosair Children's Hospital (KCH).

According to KCH Policy \#6001.1, the AEU is an admission unit whose purpose is to "efficiently and safely admit patients from physician's offices, other health care facilities, or patient's homes." More specifically, "the intent of the AEU is to register the patient, complete the Admitting Team's initial assessment, obtain a brief history and physical assessment, and initiate treatments/interventions prior to transfer to the appropriate unit." Further, the goal of the AEU is to complete the admissions process faster than if the patient were to be admitted through the emergency department. Currently, the process of admitting patients through the AEU has not met the expectations of the $\mathrm{KCH}$ administration. Exceeding the emergency department time can be partially attributed to the fact that patients who are referred to $\mathrm{KCH}$ for admission can enter the system by one of seventeen different ways. No two ways of entering the system are standardized. 
From a patient standpoint, when the patient arrives to the hospital, the patient can be admitted in a variety of different units depending on several factors, including day of week, time of day, capacity, request of physician, etcetera. This type of variation can lead to added stress or frustration for the patient, parent or guardian, as well as additional risk to the patient in the form of delays. Historically, delays in admissions for the AEU patients have resulted in resuscitation and/or a change to a higher level of care in more than one instance.

\subsection{Research Objectives}

The purpose of this research was to evaluate and provide recommendations to reengineer the current direct admission process for the AEU of Kosair Children's Hospital to help expedite the admission process. It is understood that this was a lofty goal that may not be achievable in just one research study. With that in mind, objectives were established that when completed, would aid in completing the overall goal of evaluating and reengineering the current process. The first objective of this research was to collect and evaluate all written documentation the staff uses with the intention of standardizing the patient data collected for patient admissions. The second objective was to define the current status of the admission process from a patient flow, resource availability, and information standpoint. The third and final objective of this research was to define the problem areas of the admission process and provide recommendations for improvement.

The first objective involved collecting any written protocols, training material, forms, or standing orders that are used by the staff of $\mathrm{KCH}$. Evaluation of these documents elaborated on the written policies and procedures. For example, they showed 
which personnel were responsible for collecting specific information required for admission.

The second objective was to define the current status of the AEU from a patient flow, resource availability, and information standpoint. To accomplish this, direct observations and interviews with personnel were performed in the AEU to see firsthand how the AEU functions. To define the patient flow, observations were performed from three points of view, 1) the patient's point of view, 2) the nurse's point of view, and 3) the doctor's point of view. For the patient observations, the observer followed the patient from the time he/she entered the AEU until the time they left the unit. For the nurse and doctor observations, the observer followed an AEU nurse and doctor as he/she went about their daily routine. Also, to meet this objective, interviews were performed with the administrators, unit managers, doctors, nurses, and staff.

The third objective of this research was to identify the problem areas of the admission process based on findings from the observations and interviews and provide potential solutions to the problems. The problem areas and potential solutions will be presented to Kosair Children's Hospital administrators and staff.

\subsection{Research Significance}

The goal of healthcare is to treat and care for patients. The effectiveness of healthcare organizations in reaching this goal is evaluated based on patient outcomes. A significant factor to patient outcomes is the timeliness of administering a specified treatment. Lengthy admission processes delay treatments to patients which in turn have the potential to negatively impact patient outcomes. Hence, it can be reasonably assumed that there is a direct relationship between timeliness of admission to treatment 
effectiveness to patient outcome. Therefore, undertaking process improvements to consistently meeting the goal of a decreased time of admission standard can be directly attributed to the goals of healthcare and the best interest of patients, i.e. patient safety.

\subsection{Dissertation Organization}

This dissertation was written in manuscript format and is organized as follows. In Chapter 2, a literature review is presented, including literature pertaining to research performed on admission units, frameworks used to analyze systems and different techniques to collect data. Chapter 3 is entitled, Workflow Failures and Recoveries in an Admissions Unit, and focuses on identifying failures with in the AEU, the amount of time required to recover from the failures, and identifying the major area of the process where failures are most prominent. Chapter 4 is entitled, How an Employee's Job within an Express Admissions Unit Affects their Perception of the Workflow, and focuses on the ability of personnel in the AEU to create a process workflow of the AEU and their ability to identify all the steps in the process, even those not performed by them. Chapter 5 is entitled, Assessment of a Rapid Admissions Unit for Redesign, and focuses on providing problem areas in specific AEU process steps and potential recommendations to improve those processes. Lastly, Chapter 6, conclusions and future research will be discussed. 


\section{CHAPTER 2 LITERATURE REVIEW}

Hospital admission processes have long been identified as an area for potential improvement related to patient safety. However, in the literature, no one method/approach to admissions is agreed upon (Walker \& Haslett, 2001; Keenan, Doig, Martin, Inman, \& Sibbald, 1997). Additionally, most published research present specific case studies grounded in the methods and procedures related to a specific hospital system or organization. Thus, it is very difficult to compare and contrast the different methods in the literature based on the differences in the organizations in which the research is being conducted.

It is well known that hospitals are some of the most highly variable work environments. This type of environment lends itself to errors that can potentially lead to unintended consequences to the patient, ultimately resulting in diminished patient outcomes. In the admission process, these errors can occur in a number of places, such as medication reconciliation, obtaining past medical history, and assignment of severity level. An example of how prevalent some of these errors are can be seen in one study where unintended errors were examined by comparing medication admission information to the patient's comprehensive medication history. Of 151 patients observed, 81 patients had at least 1 unintended discrepancy (Cornish et al., 2005).

There are many different techniques researchers use to evaluate variation in healthcare systems; Prospective Risk Assessment (PRA) which include the techniques Failure Modes and Effects Analysis (FMEA) and Fault Tree Analysis (FTA), Lean 
techniques which includes process mapping, Root Cause Analysis (RCA), Systems Engineering Initiative for Patient Safety (SEIPS), etcetera. Each technique has a different method or purpose to identify variation or reason for variation. For example, RCA is a reactive process used for identifying the basic or causal factors that underlie variation (Joint Commission, 2004; Latino, 2004) and PRA is a process that examines events that contribute to adverse outcomes through the use of event tree analysis and FTA (Wreathall \& Nemeth, 2003). This research will implement two of the above mentioned techniques; SEIPS and process mapping, along with direct observational data collection and interviews with the staff and personnel who work directly with the AEU.

\subsection{Systems Engineering Initiative for Patient Safety}

When attempting to redesign any system, an important process that needs to be performed is to use a model to break down the system into its elements. Doing so allows the researcher to see what completely makes up that system. However, as stated by Carayon et al. "noticeably missing from the patient safety literature are models to guide studies to empirically examine system design in relation to patient safety" (2006). With this lack of models, Carayon et al. (2006) created the Systems Engineering Initiative for Patient Safety (SEIPS) model. The SEIPS model goes further than other models by specifying the system components that can contribute to causes and control of medical errors, incidents, and adverse events. This model also has the ability to show the nature and design of interactions between components contributes to acceptable or unacceptable processes (Carayon et al., 2006)

The structural characteristics of the SEIPS model uses a five point work system to evaluate the system processes/interaction between hospitals, primary care clinics and 
patients and caregivers based on patient safety outcomes, Figure 1. A basic description of the five points of the work system is:

- Technology and tools include items such as electronic health records, templates, forms, medication lists discharge summaries, etc.

- Environment refers to the physical layout of the work spaces, work culture, atmosphere, etc.

- Tasks include the procedure or objective

- Organization represents team structures, the policies and procedure, relationships with hospitals, etc.

- People refers to anyone who interacts based on the other four points: healthcare providers, patients, caregivers, family, etc.

The SEIPS model provides insight on the complexity of the system and the many interactions between the different elements of the system and provides focus on all aspects of the work system, not just certain aspects such as people or tasks. 


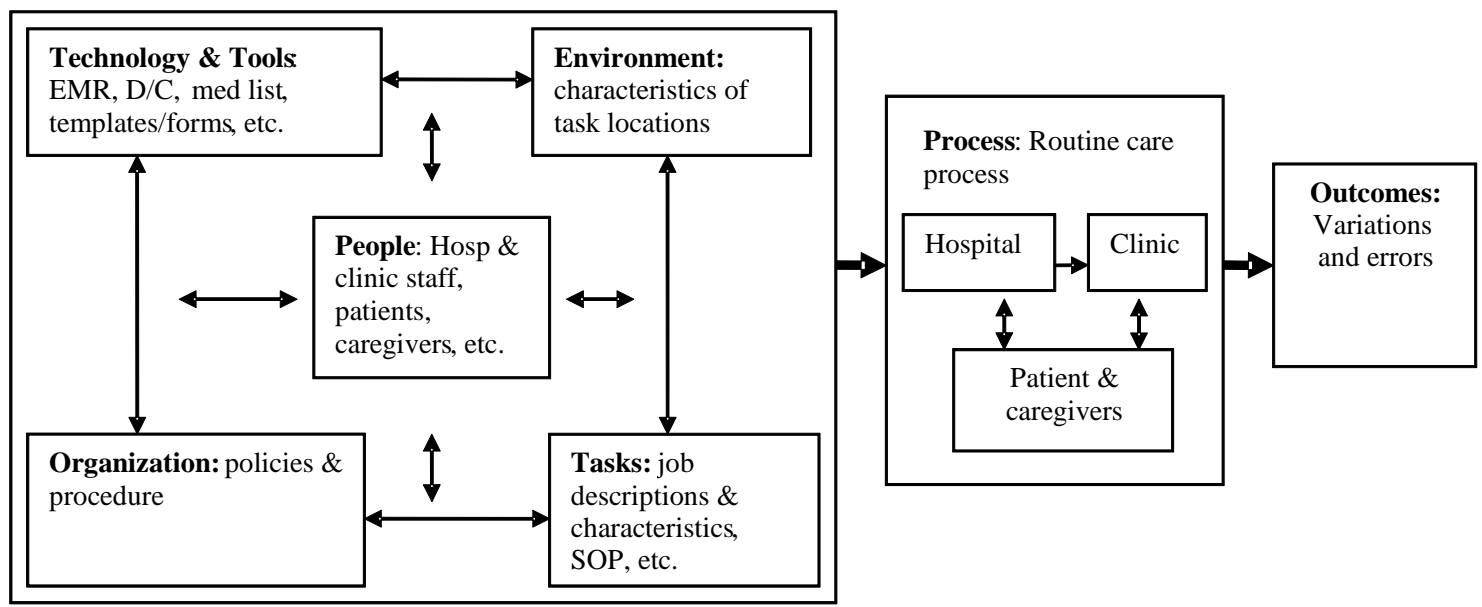

Figure 1: SEIPS Model

Since the creation of the SEIPS model, it has been used in a variety of ways to analyze and breakdown systems. Hysong et al. (2009) used the SEIPS model to analyze Electronic Health Record (EHR) implementation in an outpatient setting while Hoonakker, Cartmill, Carayon, and Walker (2011) used SEIPS to evaluate the implementation of EHR in Intensive Care Units. Carol Boston-Fleischhauer proposes combining Human Factors Engineering (HFE) and Reliability Engineering to improve healthcare process design (2008). Using the SEIPS model for HFE and reliability design methods for Reliability Engineering, she proposes that the application will better position organizations to optimize the results of important process design and implementation efforts (Boston-Fleischhauer, 2008). Since the SEIPS model examines system design in relation to patient safety and breaks the system down into its main components, it was selected as a tool that would be helpful in this research. 


\subsection{Process Mapping}

Process mapping is one of many techniques that fall under Lean thinking. Lean thinking is based on the philosophy created by Toyota Motor Company which focuses on minimizing the total time and resources required to produce goods or provide service to customers (Mazzocato et al., 2012). Process mapping has successfully been adapted from being used in industry to being used in healthcare. Trebble et al. describes very well what should be found and used while process mapping the patients journey through a hospital (2010). They state that the process map should comprehensively represent the patient journey, information relating to the steps or representing movement of information can be added, and that it is useful to obtain any missing information at this stage (Trebble et al., 2010).

As stated, process mapping has been successfully used to improve healthcare systems. For instance, King, Ben-Tovim, and Bassham used process mapping and were successfully able to group patients into groups, those who are likely able to return home and those likely to be admitted to the hospital. This in turn allowed the patients to be cared for by a specific team of doctors which helped decrease the potential for overcrowding (2006). Another study performed by Johnson et al. used clinical teams from six countries to create a process map and determine the missing pieces during care transitions (2012). The process map showed them similar barriers to providing information to primary care physicians, inaccurate or incomplete information on referral and discharge, problems with collaboration with counterpart colleagues, and lack of feedback to clinicians involved in the handovers (Johnson et al., 2012). 


\subsection{Observation and Interview Techniques}

There are many different techniques for doing research in healthcare. For example, there are retrospective methods such as review of patient records or review of previously collected data. There are also prospective methods such as surveys, nonparticipant (non-direct) or participant (direct) observations, focus groups, or one-on-one interviews. Each method has successfully been used in the literature. For instance, Mazzocato et al. used non-participant observations to collect data that was used to implement Lean techniques in an emergency care setting (2012). Focus group interviews were performed in six academic health centers around the world in a study aimed to demonstrate how process mapping could 1) illustrate handover practices between ambulatory and inpatient care settings, 2) identify barriers and facilitators to effective

transitions of care, 3) and identify areas for quality improvement (Johnson, et al., 2012). Two studies performed by Wiegl et al. dealt with analyze the effect of interruptions on doctors and their workflow (January 2011, December 2011). In both of these studies, the direct observation technique was used to observe doctors' work shifts.

As can be seen, these methods have successfully been used to analyze work systems within healthcare. For this research study, the techniques of direct observation and one-on-one interviews with the methods, as described by John Creswell in Research Design (2009), will be used. Creswell explains that the direct observation steps include:

- Identifying the individuals to participate

- Indicate the type of data to be collected

- Take field notes in an unstructured or semi structured way 
- Determine role the researcher will take varying from non-participant to complete participant (2009).

For one-on-one interviews, Creswell explains that one should develop a protocol for asking questions and recording answers and should include:

- A heading

- Instructions for the interviewer

- Questions and probes for each questions

- Space between each question to record responses

- A final thank-you statement (2009).

Finally, Creswell explains that for both observation and interviews, not only can hand written notes be used but also audiotaping or videotaping can be used to take notes (2009). 


\section{CHAPTER 3}

Workflow Failures and Recoveries in an Admissions Unit

\subsection{Introduction}

The admission process is the first phase of care for most patients who are referred to a hospital for admission. The admission process generally consists of gathering patient information and medical history, performing a brief physical examination, and registering the patient which includes placing a personal identification bracelet on the patient, and having the patient sign general consent forms, so treatment may be performed. While this is the norm for most hospital admission processes, variations exist between hospitals relative to how these tasks are performed and in what order. Such is the case with the Admission Express Unit (AEU) of Kosair Children's Hospital (KCH). The AEU is a designated unit for doctors or other hospitals to send low to moderate pediatric patients for direct admission to the hospital without having to go through the emergency department.

Processes change over time, adapting to new treatment methods and situations, and this is especially true in healthcare. Recently, administrators of Kosair Children's Hospital expressed concern with the amount of time it now takes patients to complete the Admission Express Unit and be roomed. This concern was raised after several adverse events occurred to patients that were attributed, in part, to delays in admission. One event required resuscitation to be performed. Further, upon evaluation, the $\mathrm{KCH}$ administration stated that the purpose of the AEU had changed since its creation in the 
1990 's. Its original intention was to quickly assess the patient, start the patient's paper work, perform any labs, IV's, or x-rays, and then transfer the patient to the appropriate type of room. However, over the years, additional steps, such as initial treatment of patients and consultations by specialist have been added to the AEU process. These additional steps have prolonged the process of admitting the patients that, in turn, has hindered the original purpose of the AEU.

Not only did the additional steps add more to the overall time to get through the process, but like any process, the AEU is impacted by events and system failures that result in delays in care. With that in mind, the objective of this study was to evaluate the AEU for events and systematic failures that cause delay and to determine the amount of time required to recover.

\subsection{Methodology}

Hospital admission processes have long been identified as an area for potential improvement related to patient safety. According to Ahluwalia and Marriott, "approximately $10 \%$ of all hospital admissions are complicated by critical incidents (events) in which harm is caused to the patient" (2005). However, in the literature, no one method/approach to admissions is agreed upon (Walker \& Haslett, 2001; Keenan, Doig, Martin, Inman, \& Sibbald, 1997). Additionally, most published research has been specific case studies grounded in the methods and procedures related to a specific hospital system or organization. Thus, it is very difficult to compare and contrast the different methods in the literature based on the differences in the organizations that the research is being performed. 
Approval was given by the University of Louisville's and Norton Healthcare's Internal Review Board (IRB) in May 2012. For this study, data was collected in two steps. First, observational data was collected from the patient's point of view. Since process redesign starts with the patient-eye view (Ben-Tovim, Dougherty, O'Connell, \& McGrath, 2008) and the patient is the objective and purpose of any healthcare system, it was important to view the process steps from the patient's point of view. This allowed the researcher to view the process as if they were the person being treated, i.e. experience all the processes and delays a patient must go through while in the AEU. Secondly, observational data was collected from the care provider's point of view. Tandem observations were to be implemented by two observers, one researcher observing the nurse and the nurse's point of view while the second researcher was observing the doctor when he/she entered the AEU until he/she left (Wetterneck \& Holman, 2011). However, once observations were started, it was determined that the physicians spent significantly less time in the AEU as compared to the nurses. Hence, for this study, it was decided that observations from the nurse's point of view was more relevant to the goal of the study, since, they were observed as taking ownership of the patient and having the vast majority of the contact time with the patient. The resulting time split was approximately $75 / 25$, nurse to doctor over the entire observation process.

In both steps of the data collection, data was collected in the form of hand written notes and audio tape. Hand written notes were taken while observations were taking place along with an audiotape recording of all conversation the patient had with any doctor, nurse, or staff member of $\mathrm{KCH}$. The audio recordings were transcribed anonymously and combined with the hand written notes to create a complete set of notes 
for each patient visit which was analyzed in the NVIVOC software package (QSR International).

All doctors, nurses, and staff members of $\mathrm{KCH}$ were asked to volunteer to be observed and signed a consent form. All patients were verbally asked to consent to be observed. If anyone declined to participate or asked to quit being observed at any point during the observation, the observer turned off the audio recording, stopped taking notes, and left the patient's room immediately. Observers were trained in evaluation techniques to record the process while not interacting with it. All observers adhered to hospital protocols. No one except members of the research team evaluating the procedure had access to raw data.

\subsection{Results}

Initial data collection occurred from May 2012 to July 2012. One hundred and ten hours of observation over 14 days were performed in the AEU to collect 20 patients. Staff tasks and interactions were observed when patients were not present. It should be noted that many times, staff stated that this summer season was an unusual slow period with a below average number of patients present in the AEU. Secondly, observations from the nurse's point of view were performed from January 2013 to March 2013. Twenty-four hours of observation over eight days were performed observing the nurse with a total of $17 \mathrm{AEU}$ patient visits. This time period in $\mathrm{KCH}$ is considered peak season resulting in a high number of patient visits compared to the June Observations.

Upon completion of the observations and transcription of the audiotapes and hand written notes, a macro level flow of the AEU process was created. The macro level flow of the AEU can be seen in Figure 2. 


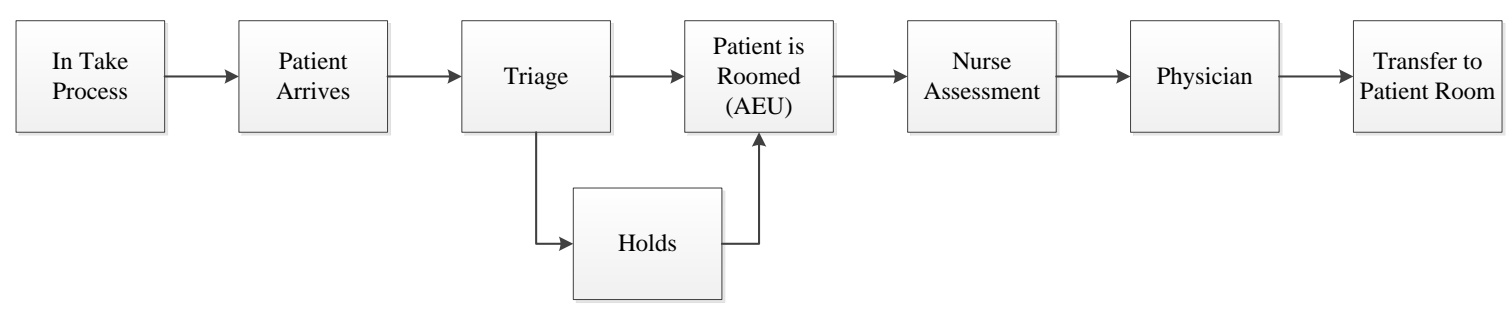

Figure 2: Macro Level Process Flow of AEU

The first step in the AEU process is the In Take Process. This process involves the patient's primary care physician calling $\mathrm{KCH}$ and letting them know that he/she is sending the patient to the hospital to be admitted and providing all the necessary information needed for the doctors of $\mathrm{KCH}$ to treat the patient. Once the patient arrives at the hospital, the patient goes through a brief triage process, after which the patient is roomed or waits in the waiting room if there are no beds available in the AEU. After the patient is placed in an AEU room, the AEU nurse performs their assessment and gathers the patient's information to be entered into the computer. Next, the physician(s) enters the AEU and perform their assessment. Lastly, in the Transfer to Patient Room step, the patient is prepared to be moved to their room.

This macro level flow was created to provide defined steps of the process that could be used to label different deviated events and failures, which led to delays, of the AEU process. With these different steps, NVIVO (C) (QSR International) was used to code the deviated events and failures from the observational data to a specific step in the process. Each event or failure was coded using one of the five major macro level process steps or labeled as Other:

- In Take Process

- $\quad$ Triage

- Nurse Assessment
- Physician

- Transfer to Patient Room

- Other 
A deviated event or a failure was defined as any unnecessary task performed by a staff member, miscommunication or lack of communication between two people, or anything that added any unnecessary time to a process. Table 1 provides a list of examples of the defined types of deviated events or failures. Using this definition, 71 deviated events and/or failures were discovered from the 22 days of observation of both the patients and the nurses of the AEU.

Table 1

Examples of Deviated Events or Failures

\begin{tabular}{|c|l|}
\hline Type & \multicolumn{1}{c|}{ Example } \\
\hline Unnecessary Task & $\begin{array}{l}\text { Nurse asks Aid for vitals when vitals were on } \\
\text { slip of paper next to nurse }\end{array}$ \\
\hline Miscommunication & $\begin{array}{l}\text { House Manager forgot to tell the AEU nurse } \\
\text { what room patient was going to }\end{array}$ \\
\hline $\begin{array}{c}\text { Added Unnecessary } \\
\text { Time }\end{array}$ & $\begin{array}{l}\text { Nurse has to go get numbing agent from the } \\
\text { emergency department because AEU was out }\end{array}$ \\
\hline
\end{tabular}

Each deviated event or failure that was found was put into one of the five process categories or labeled as Other. A complete listing of each event or failure and where in the process they occurred can be found in Appendix A. Each listing shows the amount of time that was required to recover from each deviated event or failure and a classification for each event or failure in terms of the type, i.e. communication, equipment or supplies, etcetera. Three events or failures were omitted in this table because a time to recovery (TTR) could not be calculated since the observations ended before the event or failure was resolved. Table 2 gives a summary of these results with Figure 3 providing a percentage breakdown of the number of deviated events or failures in each AEU process step. 
Table 2

Summary Table of Deviated Events and/or Failures of the AEU

\begin{tabular}{|l|l|l|l|}
\hline Process Step & $\begin{array}{l}\text { Number of Deviated } \\
\text { Events or Failures }\end{array}$ & $\begin{array}{l}\text { Average } \\
\text { TTR }\end{array}$ & $\begin{array}{l}\text { Median } \\
\text { TTR }\end{array}$ \\
\hline Intake Process & 4 & 32.0 & 29.5 \\
\hline Triage & 1 & 5.0 & 5.0 \\
\hline Nurse Assessment & 25 & 6.0 & 2.0 \\
\hline Physician* & 14 & 17.6 & 9.0 \\
\hline Transfer to Patient Room & $\wedge$ & 31.1 & 7.5 \\
\hline Other & 8 & 3.5 & 2 \\
\hline $\begin{array}{l}* 2 \text { outliers omitted } \\
\wedge\end{array}$ & & \\
\end{tabular}

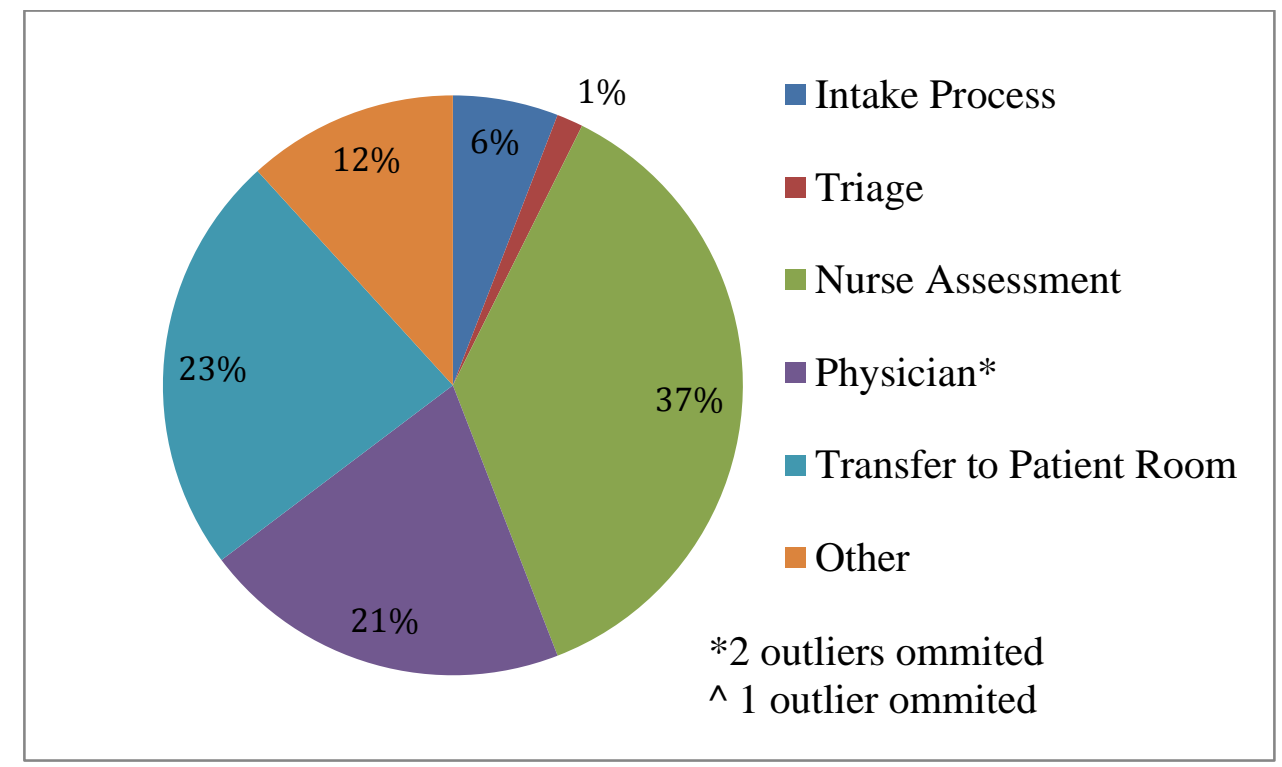

Figure 3: Percentage of Deviated Events and/or Failures in AEU Process Steps

To determine if there was a significant difference between the process steps and the amount of time required to recover from the deviated events or failures, an analysis of variance (ANOVA) was conducted. Before performing the ANOVA, since the data collected was not normally distributed, a Box Cox Transformation was applied to the TTR using Minitab. The ANOVA on the transferred TTR showed the process steps were significant at a $95 \%$ confidence level $(\mathrm{p}=.002)$, meaning there is at least one process step 
that is significantly different than the rest in terms of mean transformed TTR. To determine which step is significantly different, a Tukey's Test was performed. Based on the sampling, the Intake Process had the highest average TTR as seen in Table 2. Statistically, the mean transformed TTR for Intake Process, Physician, Transfer to Patient Room, and Triage are not significantly different.

\subsection{Discussion}

The purpose of this study was to determine where delays and failures occur within the AEU process and to calculate the time it took for staff to recover from the delays and failures. To accomplish this, observational data was collected from the patient's and provider's point of view. This data was then analyzed using the NVIVOC software package (QSR International). Table 2 provides three points about the deviated events and failures of the AEU:

1. The process step with the highest TTR was the Intake process with an average TTR of 32 minutes.

2. The process step with the second highest TTR was the Move Out and Up but the longer delays in this process are based on the hospital being at capacity, which is out of the control of the AEU.

3. The majority, $37 \%$ percent, of the deviated events or failures in the AEU is in the nurse assessment step of the process, but the TTR averages 6 minutes.

It was determined that the AEU averages about 1.1 deviated events or failures every hour in the AEU. Considering that half of the data collected was during a "slow" time period, the average number of deviated event and failures during this time was 0.78 
per hour. Likewise, observations during the "peak" time period revealed deviated events and failures occurring at a rate of 1.65 per hour.

To fully understand the significance of an average of 1.1 deviated events or failures per hour, it is important to understand where in the AEU process the events or failures occurred and the amount of time required to recover from the event or failure. The overall average time to recovery was calculated to be 15.5 minutes. To extrapolate, the AEU is open each weekday for 12 hours. Averaging 1.1 deviated events or failures per hour, there are almost 11 deviated events or failures that happen per day. If each requires 15.5 minutes to recover from, then approximately 170 minutes are spent each day recovering from errors, which is almost $25 \%$ of the day.

The study revealed four potential process steps that need further evaluation. The first step that should be examined is the Intake Process. As stated, statistically, Intake Process was not significantly different from the Physician, Transfer to Patient Room, and Triage. However, after discussion with administration and the personnel of the AEU, as well as the high TTR from errors, it was decided that Intake Process is an area of great concern. As seen in the observations, all four of the deviated events or failures that took place during the intake process dealt with the miscommunication or lack of information about the patient being sent to the AEU by their primary care doctor. The next step that needs to be evaluated more closely is the Physician step. The physicians spend a relatively small amount of time visiting the patient in the AEU, yet they committed 14 deviated events or failures and the average time to recovery for each was 17.6 minutes.

The third process that should be evaluated further is the Transfer to Patient Room step. With this process, some delays that occurred in this step were due to the 
unavailability of general medical and surgical rooms due to capacity issues. However, this does not mean that this step should be ignored. Making the administration of the AEU aware of the capacity issues shows that there are organizational factors and facility limitations that influence the operation of the AEU. The final process step that should be evaluated is the Nurse Assessment step. The nurse assessment step had the majority of the delays and failures. However, this can be partially explained by the fact that the nurse interacts and spends the most time with the patient. The observations revealed that most of the delays or failures during the nurse assessment step were either equipment/supply issues or communication issues with the aid/unit secretary. Making these delays and failures known to the staff of the AEU could help in remedying the high number of delays and failures.

The results of this study further support that the migration of the AEU from a unit that collects patient information, performs initial physician assessments, and provide any IVs, labs, or x-rays to a unit that also performs initial treatment and patient consultations has indeed increased that amount of time for the patient to travel through the unit. However, it has also showed that the Intake Process is the most prevalent area for delays.

\subsubsection{Potential Limitations}

A limitation of this study was the lack of observational data from the physician point of view. In the AEU, the physicians were expected to round on the patients within fifteen minutes of arrival. However, few patient visits met this criterion and some patients were still waiting to be seen when the observation period ended up to four hours later. However, as previously stated, it was determined that observations of the nurse assigned 
to a given patient provided more than sufficient information regarding both the patients and physicians status relative to the patient's procedures and admission.

\subsection{Conclusions}

Delays in healthcare process pose problems in hospitals today (Lane, Monefeldt, \& Rosenhead, 2000; Horwitz \& Bradley, 2009; Stone, Boehme, Mundorff, Maloney, \& Sriastava, 2010). These delays create risk for patient by preventing timely delivery of care. The purpose of this study was to evaluate the Admissions Express Unit (AEU) of Kosair Children's hospital to determine where the delays and failures occurred in the admissions process. After observing the AEU from both the patient and nurse's point of view, 71 deviated events or failures were found, averaging to 1.1 events or failures every hour. In particular, the Nurse Assessment, which had $37 \%$ of the deviated events or failures, was found to be the process step with the most delays. While this step did not contribute significantly to the time to recovery, the number of delays or failures that occurred in this step is troubling. However, of more concern, the Intake Process was found to have the highest average time to recovery at 32 minutes per deviated event or failure. A time to recovery of this significance is problematic for the patient to get through the AEU in a timely fashion considering this is the first step the patient incurs and considering that the other process steps cannot be executed until the event or failure is resolved.

\subsubsection{Future Work}

With most healthcare processes, the devil is in the details. The same can be said with the AEU process. Having only considered the macro level steps of the AEU, the next step of this study is to breakdown each macro level step into its individual steps to 
determine if the delays and failures lie within a particular step. Doing so will allow the administration of $\mathrm{KCH}$ to look at the particular steps for potential improvements. This specifically needs to be done for the intake process and nurse assessment process to determine what micro level tasks create the largest time to recoveries and the highest percentage of delays and failures. 


\section{CHAPTER 4}

How an Employee's Position Affects Their Perception of the Workflow

\subsection{Introduction}

One of the most evaluated processes in healthcare is hospital admission. Whether it has been research performed on medication reconciliation (Baker, Lindquist, Liss, \& Noskin, 2010; Stone, Boehme, Mundorff, Maloney, \& Sriastava, 2010; Unroe, Pfeiffenberger, Riegelhaupt, Jastrzembski, Lokhnygina, \& Colón-Emeric, 2010), effects on the admission unit when patients with a particular sickness, disease, or a particular age are admitted (Kafetz, 2010; Flanagan, Ellis, Baggott, Grimsehl, \& English, 2010; Simon, et al., 2010), or admission process improvement (Johnson, et al., 2012; Huang, Thind, Dreyer, \& Zaric, 2010), it is recognized as the beginning of the treatment process and, if performed efficiently, the first critical step towards improved patient outcomes. Hence, the need for improvement is always present. The challenge with research in this area is that no one method or approach to perform the research is accepted (Walker \& Haslett, 2001; Keenan, Doig, Martin, Inman, \& Sibbald, 1997). Additionally, most published research are specific case studies grounded in the methods and procedures related to a specific hosptial system or orgnazation making it very difficult to compare and constrast the different methods based on the differences in the organizations that the research was being performed in.

Kosair Children's Hospital (KCH) is a 260 bed, level 1 trauma hospital located in Louisville, Kentucky. KCH has a unique feature, for a children's hospital, in that it has a 
rapid admission unit that is called the Admissions Express Unit (AEU). When physicians determine that a child needs to be hospitalized, this unit provides a quick alternative to going through the emergency department (ED). In the AEU, the necessary information is collected, the admitting team visits the patient, and procedures, labs, or x-ray are performed. After, the patient is ready to be transferred to a patient room. This whole process is intended to expedite admission, reducing the processing time from 4-5 hours, as seen in the ED, to one hour upon patient arrival.

Since the creation of the AEU in the 1990's, the purpose of the unit has morphed from the process described above to a process that now includes consultations with specialists and the beginning of treatments; a process that now lasts 3-4 hours. With this increase in time, the adminstration of $\mathrm{KCH}$ requested an evaluation of the unit to determine areas for improvement. Direct observational data were collected from two points of view, the patient and the nurse. Upon initial observations, it was evident that the way the AEU personnel viewed the workflow of the AEU was greatly influenced by their position within the unit and this viewpoint effected the way the AEU functioned.

A review of literature found that there has been little research performed on how a person's role or responsibility affects that individual's perception of the workflow that they work in and how they perform their job to support the process. A study performed by Boan, Nadzam, and Clapp Jr. categorized four groups into different levels, frontline employees, mid-managers, senior executives, and physicians and determined whether their responses to the Agency for Healthcare Research and Quality (AHRQ) Hospital Survey on Patient Safety Culture (HSPSC) were correlated based on the differences in level of the groups (2012). Boan et al. found data showing that variance between the 
roles indicates a hierarchical barrier and that reducing that variance has an impact on the perceived quality and safety (2012). Another study wanted to examine nurse's decision making as it related to discharge planning and perceptions of their role (Rhudy, Hollan, \& Bowles, 2010). Where Rhudy et al. (2010) wanted to examine the perception that nurses had on their own role, this research wants to examine the perception that the staff of the AEU has on others' roles. Thus, the purpose of this research was to determine how an individual's role in an admissions unit affects their perception of the unit's workflow and goals, which they are tasked to support.

\subsection{Methodology}

Approval for this study was given by the University of Louisville's Internal Review Board (IRB) in May 2012. In February of 2013, ten interviews were performed with personnel of different levels associated with the Admission Express Unit at $\mathrm{KCH}$. Similar questions were asked for each group with some minor variations based on what information was needed from each position. All groups were asked the same questions in regards and to further creating the process flow of the AEU on the whiteboards from their perspective. A more detailed description of the interview process and the method of using the whiteboards is given below.

\subsubsection{Participants}

The interviews with the personnel were based on a stratified sample of doctors, nurses, and staff members that work in the AEU, (Marshall \& Rossman, 2006) along with the unit managers and the oversight (administrator). It was important to interview various doctors, nurses, and staff that are associated with the AEU as they only see the processes for which they are responsible for, and no single staff member oversees all the 
steps in the patient's journey (Ben-Tovin, Dougherty, O'Connell, \& McGrath, 2008).

Further, it was important to interview the managers and administration of the AEU as they have a general understanding of all parts of the process. A more detailed description of the persons interviewed and their intended purpose are given:

1) Unit Manager Interviews: Interviews were conducted with the overall unit manager of the emergency department (who is in charge of the AEU) along with two assistant nurse managers. The purpose for these interviews were to:

a) Learn and request the policies and procedures governing the admission procedure and information management;

b) Discuss the process of admissions and metrics used to evaluate;

c) Understand and request alternative types of data which may be available to provide information on the admission procedures and flow.

2) Doctor, Nurse, and Staff Interviews: Three doctors, two nurses, and one aid/unit secretary were interviewed. The purpose of the doctor, nurses, and staff interviews were to:

a) Obtain an understanding of variation and its effect on the overall process;

b) Determine what needs exist that were not being met by the existing admissions process;

c) Identify the role each individual had in the process.

3) AEU Oversight (Administrator): The administrator interviewed was one of several who have oversight of the AEU and was an organizational level 
patient safety officer. The purpose of the administrator interview was to understand the need for change to the AEU, obtain the unique perspective of the AEU from the individual who is also responsible for patient safety, and to obtain the point of view of the person responsible for an outcome of the unit but who does not work in the unit.

All participants were asked to volunteer to be interviewed and signed an informed consent. The time and place of the interviews were at the discretion of the individual. Interviews lasted 30-75 minutes and were audiotaped.

\subsubsection{Interview Scripts}

Three separate interview scripts were written for the interview process. The unit managers, nurses, and unit secretary all were interviewed with one interview script (Appendix C). The doctors had their own interview script (Appendix D), as well as the administrator (Appendix E). These interviews were grouped in this way to gain particular information that, based on the person's position, only he/she could answer. There were seven main topics that the interview focused on with sub-questions to support each:

1) Background information of interviewee

2) Purpose of the AEU

3) Protocol of the AEU

4) Evaluation metrics of the AEU
5) The process flow of the AEU including delays, inefficiencies, and personnel

6) Specific questions based on observations

7) An ideal system 


\subsubsection{Whiteboard}

Process mapping has been used frequently in healthcare to aid in understanding different processes. This tool allows all the steps that make up a patient's visit visible to everyone (de Bucourt, et al., 2012; Johnson, et al., 2012; Ben-Tovin et al., 2008). To aid the investigation of above topic 5 concerning the process flow of the AEU, a magnetic whiteboard was used with the different process steps labeled on magnets to map out the process, Figure 4. The process steps were created based on the observations that were performed prior to the interviews. Blank cards were also provided should there be a step that the interviewee felt was important that had been left out. Each interviewee was asked to place the cards in the order that the AEU process takes place, from their point of view. Once the interviewee was finished, they were asked to identify the personnel associated with each task, the tasks that were the major sources of delays, and the inefficient tasks. For the tasks with major delays and inefficiencies, the participants were asked to rank the top two or three delays and rank the top two or three inefficient tasks. Upon completion of each interveiw, each whiteboard was labeled for identifaction and photographed for documentation. 


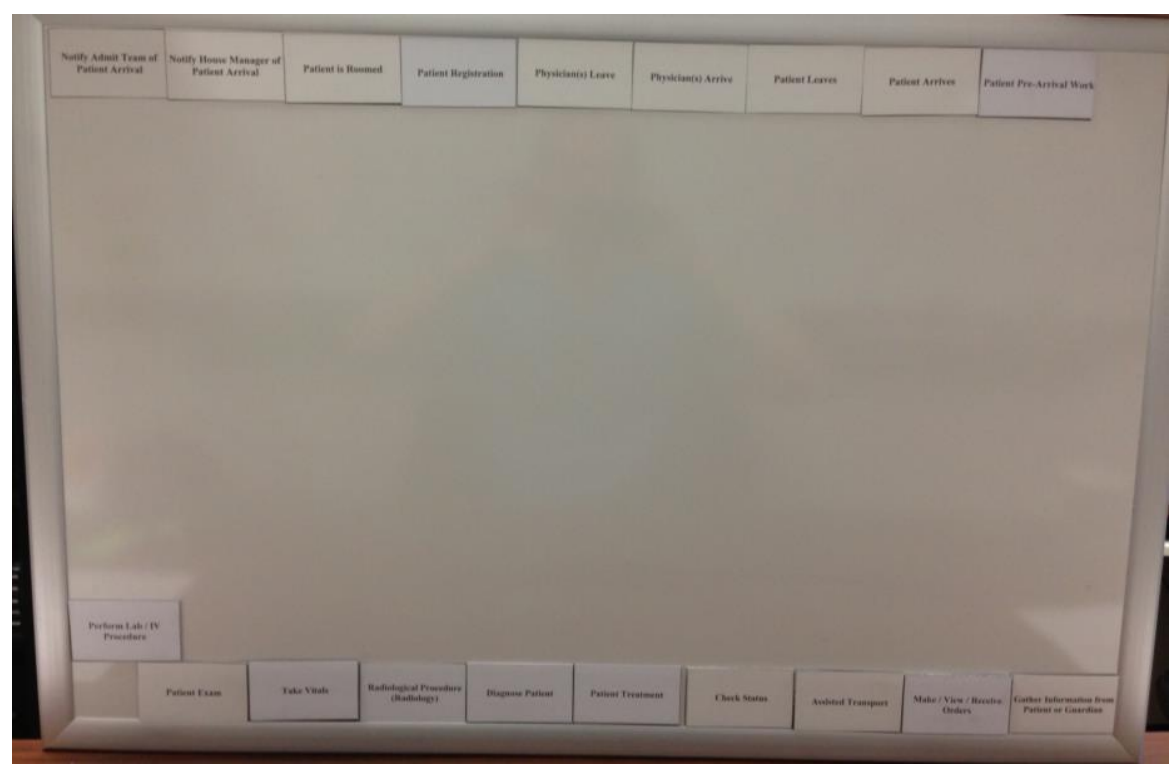

Figure 4: Magnetic Whiteboard and AEU Process Steps

After the completion of all of the interviews, the results of the whiteboards were combined into four groups based on similarity of process steps. The nurse's and unit secretary's whiteboards were combined into one workflow. The doctor's whiteboards were combined into one workflow as well as the nurse manager's. The whiteboards were combined into group workflows so that individual interviewee's process maps could not be identified. The oversight's whiteboard was not combined with any other because of the unique perspective the oversight was able to provide. Once all of the group whiteboards were created, one holistic board was created, which merged all the individual boards together. This board, like all the others, used the same format with red dots indicating delayed processes, blue dots indicating inefficient processes, and different letters indicating what people were involved in each process, as identified by the interviewees. Table 3 provides a key for the people, delays, and inefficiencies. 
Table 3

Abbreviation Meanings and Key for Workflows

\begin{tabular}{|c|l|}
\hline Letter & \multicolumn{1}{|c|}{ People } \\
\hline A & Assistant Nurse Manager \\
\hline D & Referring Primary Care Physician \\
\hline E & EMS \\
\hline F & Family \\
\hline H & House Manager \\
\hline I & IV Team \\
\hline K & Respiratory Technician \\
\hline M & Doctor, Attending, Resident, Intern, Medical Student \\
\hline N & Nurse \\
\hline O & Other (Access Center, JFK, etc.) \\
\hline P & PCA \\
\hline Pt & Patient \\
\hline R & Registration \\
\hline S & Secretary \\
\hline T & Transport (Internal) \\
\hline V & Volunteer \\
\hline X & Radiology \\
\hline O & Delay \\
\hline O & Inefficient \\
\hline
\end{tabular}

Every board followed the same format. There were five macro level steps: In Take Process, Triage, Nurse Assessment, Physician, and Transfer to Patient Room. Each macro level step was then made up of more detailed steps. Each board reads in a linear fashion from left to right. Multiple process blocks stacked upon one another means that at least one or more of these processes has to happen in this particular step.

\subsection{Results}

The nurse's and unit secretary's whiteboards were combined to form one workflow from their perspective. There were two nurses and one unit secretary. The AEU nurses and unit secretary had a total combined experience of 65 years, all at $\mathrm{KCH}$. Both nurses had been working in the AEU for approximately three years, while the unit 
secretary had been working in the AEU for approximately 13 years, which was close to the same time as the creation of the AEU. These individuals spend more time than any other personnel with the patient while they are in the AEU. They are the individuals that take vitals, collect the patient history, perform procedures, etc. Figure 5 shows the nurse's and unit secretary's combined workflow.

Figure 5 shows that the nurses and unit secretary all agreed that the process step of Physician Arriving was a major delay and a major inefficiency. All three participants from this grouping said that this process step was a major delay and two of the three in the group said it was an inefficient process. From the rankings, it was ranked as the most inefficient by one person and the third most inefficient task by another.

The individual doctor whiteboards were combined to form their collaborative work flow. For this group, three doctors were interviewed. The doctors have an average of about $2 \frac{1}{2}$ years of experience. All of the doctor's experience had been at $\mathrm{KCH}$ since their graduation from medical school and each had been rounding in the AEU since they began. The doctors spend a relatively small amount of time in the AEU. They come to the AEU after the patient has arrived and normally after the patient has been seen by the nurse. Once the doctors have performed their assessment, they provide the nurses with orders and leave the AEU. Figure 6, illustrates the combined workflow of the doctors.

There were three process steps that the doctors believed to be a major delay and/or an inefficient process and these three process steps all relate to one another. The three process steps are shown in Table 4. 
Table 4

Summary Table of Doctor's Delays and Inefficiencies

\begin{tabular}{|c|c|c|}
\hline $\mathrm{n}=3$ Doctors & $\begin{array}{c}\text { Number of Physicians that } \\
\text { said Step was a Delay }\end{array}$ & $\begin{array}{c}\text { Number of Physicians that } \\
\text { said Step was Inefficient }\end{array}$ \\
\hline $\begin{array}{c}\text { Notify Admit Team of } \\
\text { Patient Arrival }\end{array}$ & 2 & 1 \\
\hline Physician Arrives & 2 & 2 \\
\hline $\begin{array}{c}\text { Make / View / } \\
\text { Receive Orders }\end{array}$ & 1 & 1 \\
\hline
\end{tabular}

These three process steps have an additive effect on the others, meaning, that if a delay occurs in the Notify Admit Team of Patient Arrival step, then that causes the physician to arrive late and that step is perceived as being a delay. Likewise, the Make/View/Receive Orders step is delayed because the previous two steps were delayed, which in turn, actually causes the whole process to be perceived as being delayed. 

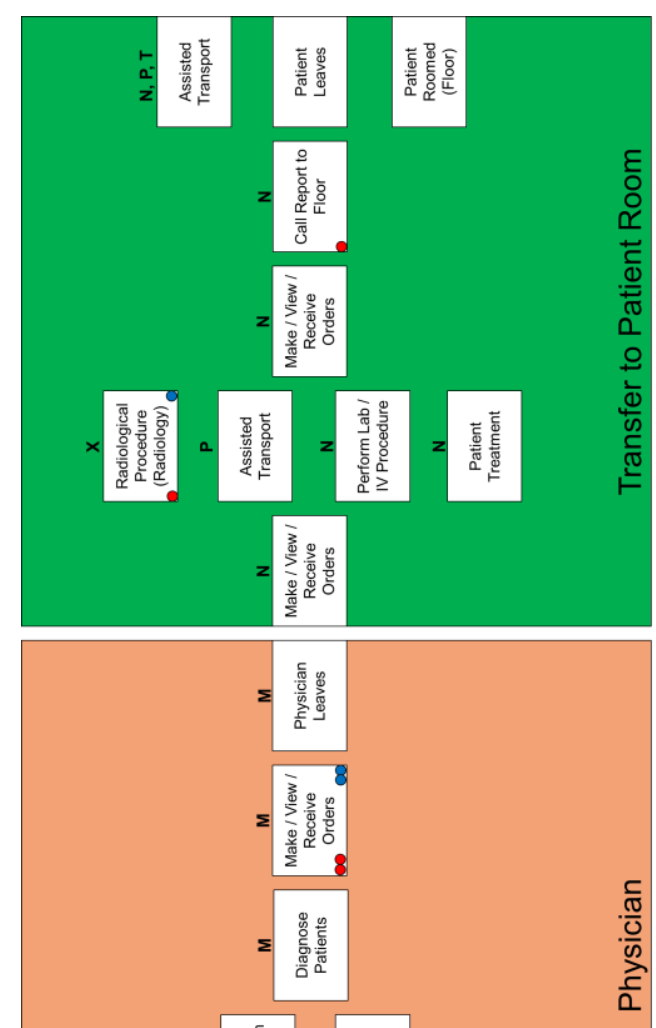

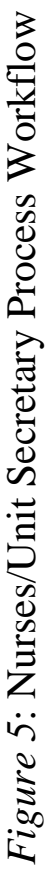

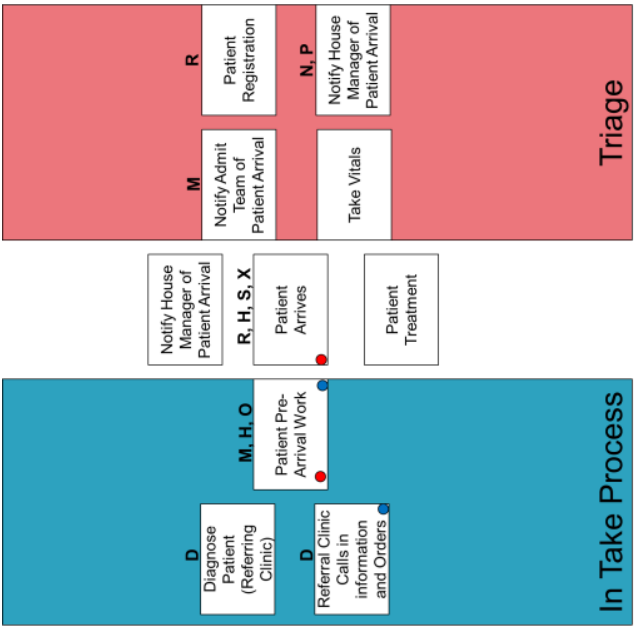



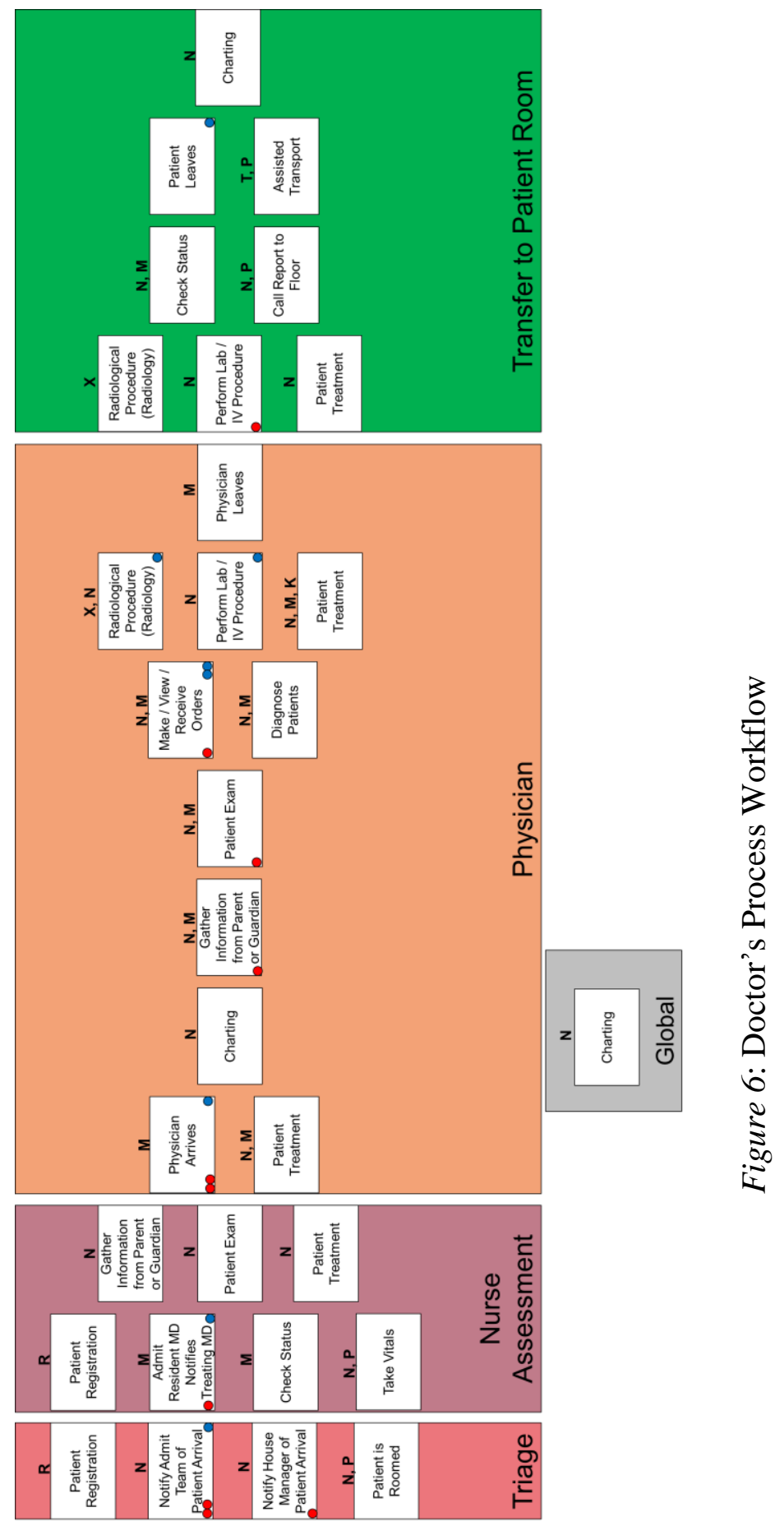

$z$ 喜这

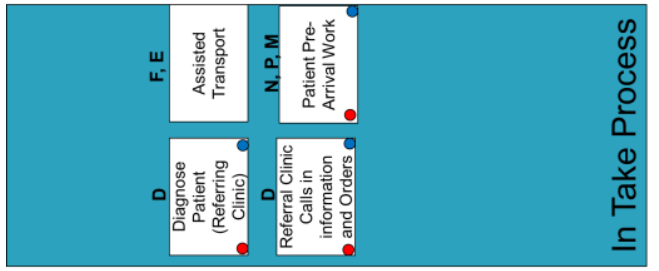


The third group of individuals whose whiteboards were combined was the Nurse Managers. Even though the AEU is not considered a part of the emergency department (ED), the nurse managers of the ED are in charge of the AEU. There were three individuals interviewed; two assistant nurse managers and the nurse manager. The nurse mangers combined had 88 years of nursing experience; however, only one of the nurse managers had ever worked in the AEU and this was only part time, for about a year. The combined nurse manager workflow is shown in Figure 7.

There was no consensus between the nurse managers concerning inefficiencies. However, there were many process steps that the nurse managers agreed were major delays. Like the nurses, all three nurse managers thought that the Physician Arriving step was a delay. Also, all three nurse managers believed that the Make/Review/Receive Orders and Radiological Procedure steps were major delays. Two of the three nurses believed that the steps Patient Roomed AEU, Perform Lab/IV Procedure, Assisted Transport, and Patient Leaves were a source of delays.

As there was only one administrator and that person had a unique perspective of the AEU, that person's process flow was kept separate from everyone else's process flow. The administrator had been a physician for 20 years but never treated patients in the AEU. Figure 8 provides the administrator's work flow. It can be seen that the administrator believed that the process steps of Patient Roomed AEU, Physician Arrives, and Call Report to Floor were major delays and there were 10 process steps that were thought to be inefficient 

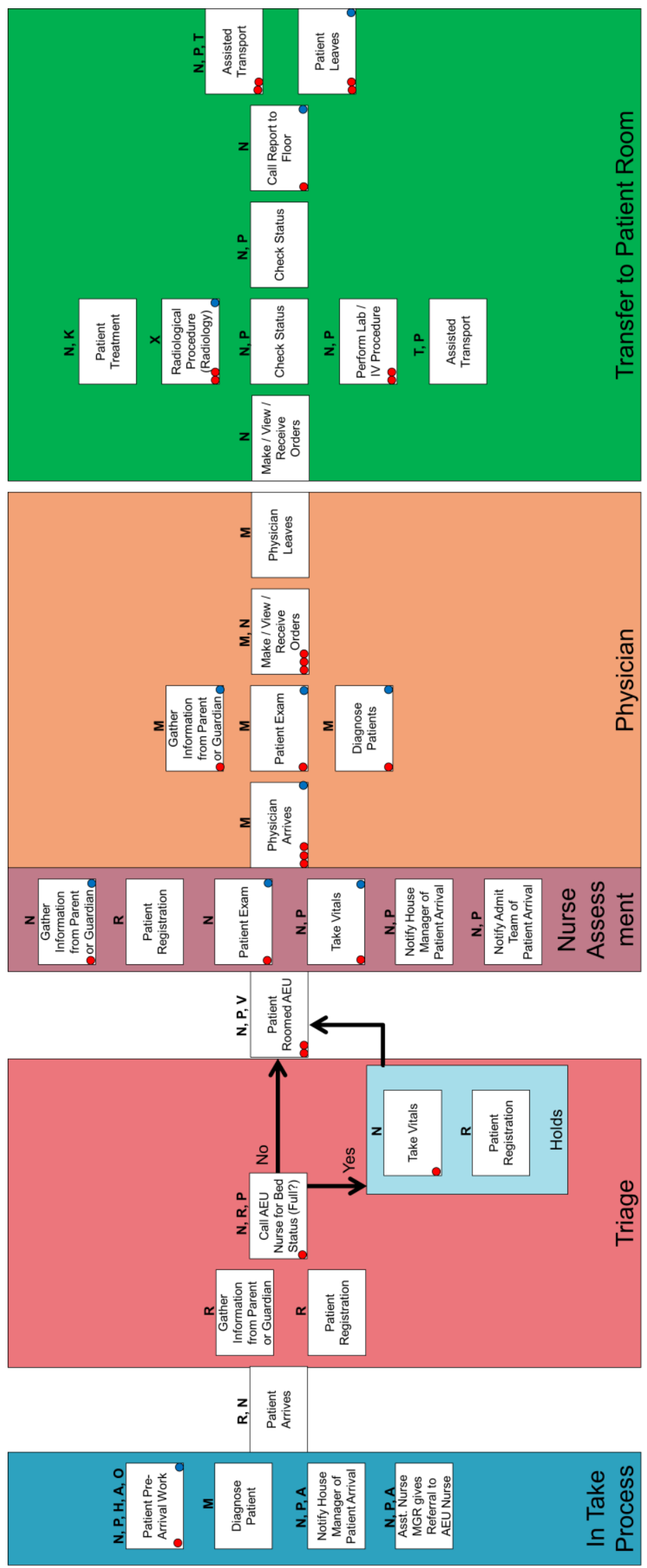

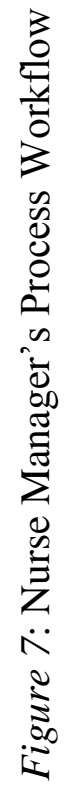




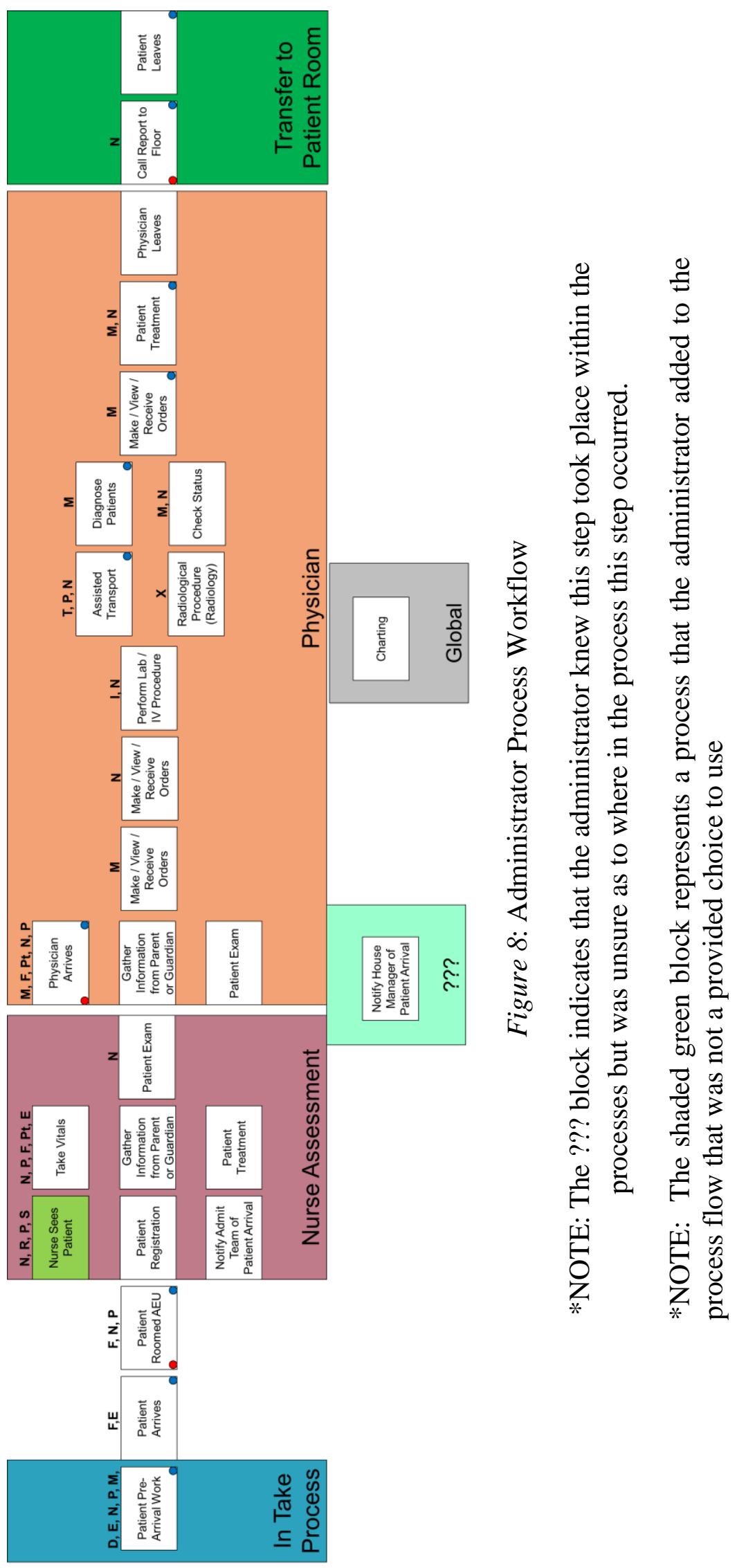


Once all of the group workflows were created, they were combined to form the overall workflow for the AEU. This flow provides an accurate representation of the complete AEU work flow, considering it contains multiple view points from all levels of personnel that work in the AEU, Figure 9. This flow was stitched together from the group flows by using key steps in the process as marker points (i.e. Patient Arrives, Patient Roomed AEU, Physician Arrives, and Physician Leaves) and placing the remaining process steps in between. Based on the responses from the groups, two process steps, Check Status and Charting, were placed above the flow indicating that these process steps were continuous and can happen at any time from the point where the process block begins to the point that it ends.

The combined flow provides telling results as to where the personnel of the AEU believe the problems are concerning delays and inefficiencies. There were five process steps that were of major concern according to the personnel:

- Patient Pre-Arrival Work

$$
(\mathrm{D}=5, \mathrm{I}=6)^{*}
$$

- $\quad$ Patient Roomed AEU (D=5, $\mathrm{I}=1)$
- Make/View/Receive Orders

$$
(\mathrm{D}=5, \mathrm{I}=5)
$$

- Call Report to Floor $(\mathrm{D}=4$, $\mathrm{I}=2)$

- Physician Arrives $(\mathrm{D}=9, \mathrm{I}=5)$

Each of these process steps had at least three people believe it was a delay (D) and inefficiency (I) or more than three people believe it was a delay or inefficiency. Consensus showed that Physician Arrives was the process step believed to be the biggest problem with 9 out of 10 personnel believing it was a major delay. It should be noted, the Patient Pre-Arrival Work step includes the delay and inefficiency count of the 
Referral Clinic Calls in Information and Orders and Diagnose Patient (Referring Clinic) steps because these two particular steps were written in by one of the interviewees and it is believed that the remaining personnel being interviewed believed these two and the Patient Pre-Arrival Work step to be the same thing. 


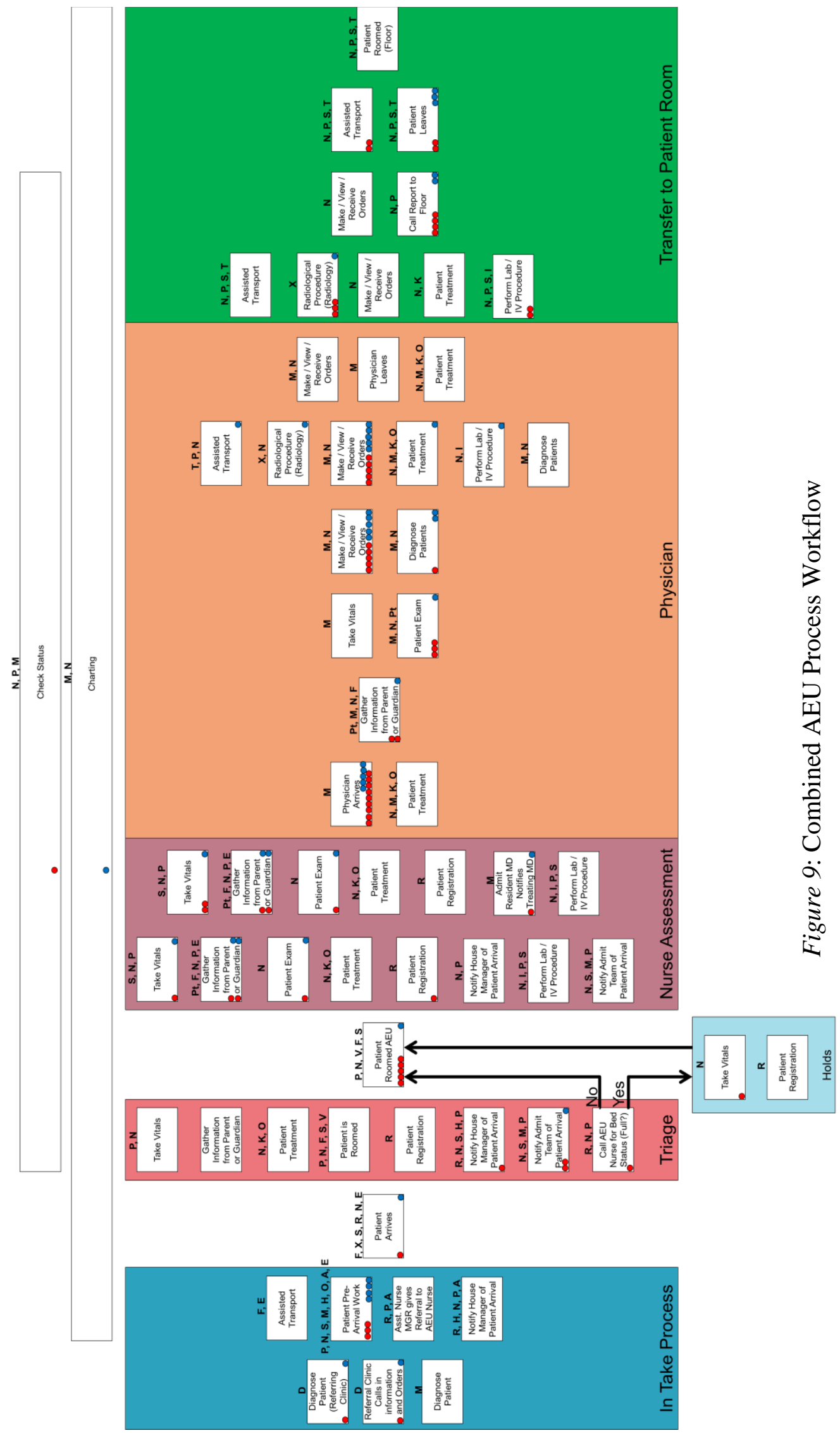




\subsection{Discussion}

The purpose of this research was to determine how an employee's position affects their viewpoint and perception of the workflow as it relates to the Admission Express Unit of Kosair Children's Hospital. The interviews with the 10 different individuals who work within the AEU provided a vast wealth of information that was used to evaluate this research question. Three observations related to perception of the workflow resulted from this evaluation:

1. The high level of detail provided about the workflow relative to the person's position within the AEU process,

2. Delays, inefficiencies, and personnel involved were marked on process steps or individuals that are important only to that person, and

3. No individual lays claim to the patient before the patient arrives in the AEU.

\subsubsection{Workflow Detail}

It was important in this research to get to the greatest level of detail to truly capture the AEU workflow. It was interesting to notice that once the group process flows were created, the level of detail the different groups were able to provide to the AEU process work flow actually followed a pyramid format, Figure 10. The top of the pyramid, the oversight (administrator), provided the least amount of detail. The next level, the nurse managers, provided more detail than the oversight. The doctors were the third level with the nurses being the bottom level and providing the most detail to the AEU process flow. Not surprising, this pyramid format is also representative to the amount of time each group level spends with the patients of the AEU; starting with the 
administrator who spends practically no time with the patients, to the nurses who spends the most time with the patients in the AEU.

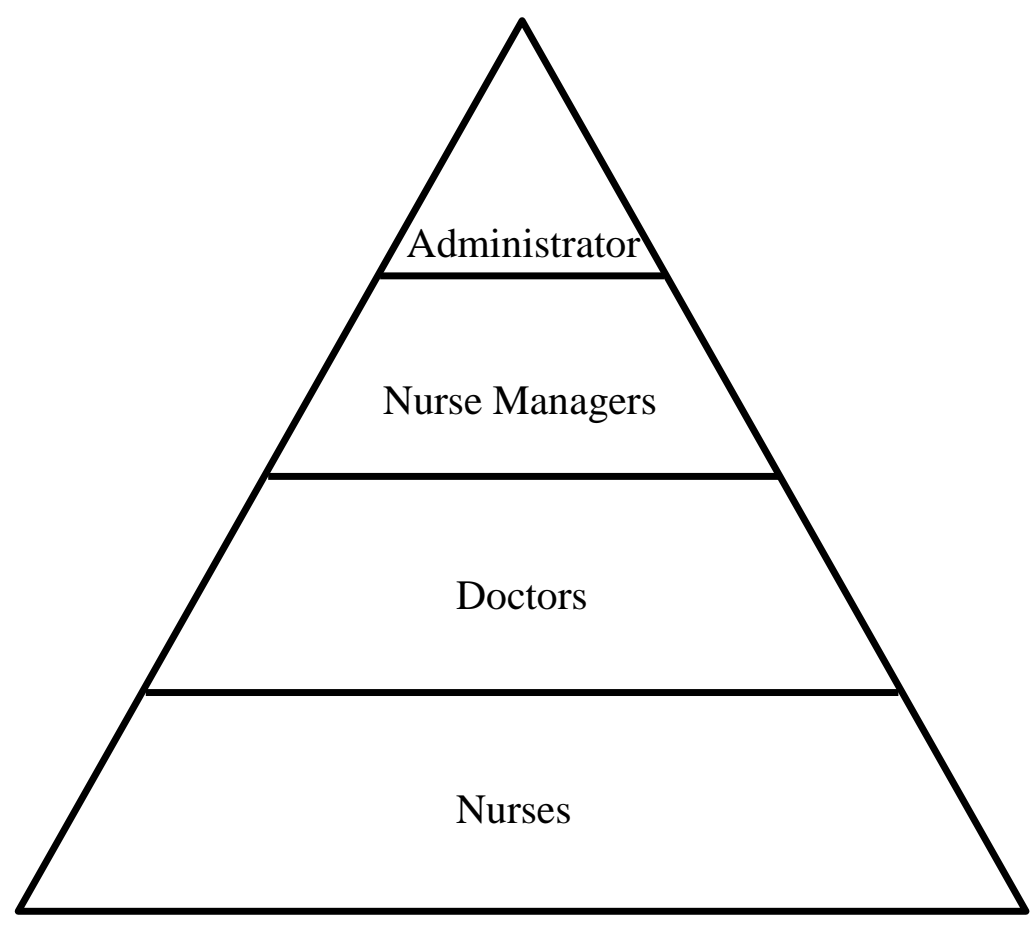

Figure 10: Representation of Detail Provided

Concerning the two groups who spend the most amount of time in the AEU, the doctors and nurses, each group provided the most amount of detail only to the process steps that they participate in. It was a common response from the doctors, when asked to order the process steps that take place before they arrive in the AEU, to say they "thought" it went this way or "I think this step happens next." However, when it came time for the doctors to order the process steps that they participated in, they were very methodical and precise in their responses. The reverse was true for the nurses. They were able to provide detail to the steps before the doctors arrived in the AEU and after they left because this was when they were involved in the process of getting the patient through the AEU. The nurses gave insight to the process steps that happen while the doctor is there, but the detail they provided was not as great as the detail provided by the 
doctor. This shows the importance of interviewing different people at different levels who are involved in a process to ensure that all steps of that process are made known with detail.

\subsubsection{Importance of Delay, Inefficiencies, and Personnel to a Group}

The second observation that came from this research was what individuals marked as delays, inefficiency, or personnel needed were important only for that particular group of people. This observation was seen across all of the groups of personnel that were interviewed. For instance, the oversight, who is also the head of patient safety for the hospital, was the only person out of the 10 individuals interviewed that included the patient and their families as individuals involved in particular process steps. As the head of the patient safety group, the oversight is mostly concerned with the patient, thus the administrator incorporates the patient into the workflow created.

The nurse managers brought an interesting perspective to the workflow because while they oversee the day to day operations of the AEU, they are really managers of the emergency department (ED). Being managers of the ED, their perception of where some delays and inefficiencies were within the AEU were the same, however, some were different than those of the nurses who work in the AEU every day. There were two main areas the nurse managers identified as problems, each of which relates back to their role as a manager. The first was the scenario of patients being placed into a holding area if there are no rooms available for them within the AEU. When there are no rooms available in the AEU, it falls on the responsibility of the nurse manager of the ED to tell the AEU nurse if they are able to place a patient in the ED to be cared for as an AEU patient. The second area that the nurse managers were concerned with was the 
Radiological Procedure step. One nurse manger stated in the interview that the ED always has to deal with delays with radiology and constantly having to call to see if they are able to bring a patient over. While the interview was concerned with the AEU, the nurse manager still related this delay to their own experiences within the ED.

The responses from the three AEU nurses/secretary demonstrations this concept of marking delays, inefficiencies, and personnel that are relative to that particular group. Looking at the flow chart created by the nurses/secretary, Figure 5, it shows two steps that they identified as main concerns; the Physician Arrives and Make/View/Receive Orders. It is interesting is that these two steps were identified as problems since both steps rely on the physician to be completed and the nurse cannot continue on in the process until these steps are completed.

Likewise, the doctor's workflow identified delays and inefficiencies with the Notify Admit Team of Patient Arrival step, which is a step that mostly involves the physicians. The doctors also identified and speak frequently about the Admit Resident MD Notifies Treating MD step and how this step and the Notify Admit Team of Patient Arrival become a big source of delay because of the need to answer and return pages and phone calls, which is another example of process steps identified that are important to a particular group.

\subsubsection{Claim to the Patient}

The final observation that was made during this research was that not one individual/group lays claim to the patient before arrival to the AEU, i.e. who has responsibility for (owns) the patient while in the care of the hospital. The fact that the admitting physician is required to call three different individuals at Kosair Children's 
Hospital to send a patient to the hospital, there is no cohesion as to who "owns" the patient until the patient arrives within the AEU's physical space. It is believed that this is part of the reason why there are so many delays and inefficiencies marked on the overall flow for the Patient Pre-Arrival Work and the Patient Roomed AEU steps. The fact that there are at least three people involved with the process of gathering the patient information when they are called in, there is a high probability that some of that information is not relayed to the necessary people leading to a delay.

Looking at the combined flow in Figure 9, many of the process steps, before the patient is placed in a room in the AEU, show a large number of possible people that could be involved compared to the remaining process steps. This further supports the observation that no individual owns the patient at this point in the process and further illustrates the need for one person to claim the patient as their responsibility until they arrive in the AEU and become the responsibility of the AEU nurse. Doing so would allow one person to coordinate what needs to be done before the patient arrives and to distribute the proper information to the appropriate people to ensure everyone has the necessary information.

\subsubsection{Potential Limitations}

A potential limitation to this research is the possible bias to responses to some of the interview questions based on the time period during which the interview was performed. The interviews were performed during the peak season of the hospital resulting in the hospital being constantly full, which in turn, resulted in the numerous patients being placed as holds within the AEU. Responses to questions like identify 
where the delays occur within the process could have been influenced based on the conditions of the hospital at that time.

\subsection{Conclusions}

The purpose of this research was to determine how an individual's role in an admissions unit effects their perception of the unit's workflow and goals, which they are tasked to support. There are five key points that can be taken away from this study:

1. The perception of where delays, inefficiencies, and personnel who perform each task differed between the different groups interviewed

2. There was consensus between the interviewees that five process steps are of major concern: Patient Pre-Arrival Work, Patient Roomed AEU, Physician Arrives, Make/View/Receive Orders, and Call Report to Floor.

3. What was marked as delay, inefficiency, or personnel responsible for the task was heavily influenced by what was important to that particular individual

4. The perception of the number of people that can potentially perform particular tasks before the patient enters a room in the AEU shows the need for one individual to claim responsibility for the patient

5. The importance of gaining insight about the workflow at different levels to overcome individual perception, thus providing a complete picture of what truly happened.

\subsubsection{Future Work}

The creation of a detailed workflow as discussed in this paper lends to the creation of a discrete event simulation (DES) model of the Admission Express Unit. DES modeling has been used successfully in healthcare to model different processes and evaluate numerous metrics including, wait time, patient throughput, resources allocation, 
staffing schedules, etc. The AEU could benefit from the creation of a simulation and evaluation of the previously mentioned metrics. Also, a simulation model could potentially illustrate to hospital administration the different delays and inefficiencies mentioned in this research to further support the need to address these areas for improvement. 


\title{
CHAPTER 5
}

\author{
Assessment of a Hospital Rapid Admissions Unit for Redesign
}

\subsection{Introduction}

According to the Institute of Medicine's (IOM) report To Err Is Human: Building a Safer Health System, as many as 98,000 people die each year as a result of preventable medical errors (1999). Ahluwalia and Marriott state that approximately $10 \%$ of all hospital admissions are complicated by critical incidents in which harm is caused to the patient, which equates to more than 850,000 incidents annually (2005). A critical incident is defined as any event or circumstance which could have or did lead to unintended or unexpected harm or injury, loss or damage (Ahluwalia \& Marriott, 2005).

When patients are admitted to the hospital by transfer from another hospital or a referral by their physician, the admissions process is typically the first process they experience. The hospital typically requires the patient's personal information and medical history, performing a brief physical examination, and registering the patient which includes having the patient sign consent forms for treatment and placing a personal identification bracelet on the patient. However, there are some hospitals that have specialized admissions units that provide alternative methods to be admitted to the hospital with the intent that the process will be streamlined and take less time. Such is the case of Kosair Children's Hospital's (KCH) Admission Express Unit (AEU). 
The AEU was created in the 1990's for patients who were being transferred from another hospital or referred by a physician. The idea was, if the patient was stable and the $\mathrm{KCH}$ nurses and doctors had immediate access to the patient's information, history, and diagnosis, the patient rooming would only be delayed by getting the necessary labs, IVs, and/or X-rays performed. At the time, this idea seemed to have merit, given the alternative of going through the lengthy admit process in the emergency department. However, over the years, the intended purpose of the AEU has changed and competing goals have been introduced into the unit. Goals such as starting treatment(s) for the patient and having consultations with specialists prior to rooming, have all delayed the unit's original intent. Further, these increased times to get through the AEU have been attributed to adverse changes in patient status, at least one case requiring resuscitation and others a change to a higher level of care, which is a major emphasis of a need for change by the Institute of Medicine (IOM) (1999). Further, this has prompted a need for change starting with the administration of $\mathrm{KCH}$ reviewing the AEU. Hence, this research was requested by $\mathrm{KCH}$; to perform an assessment of the $\mathrm{AEU}$ and to provide recommendation for the admissions process.

\subsection{Methodology}

Permission was granted for this research by the University of Louisville's Internal Review Board (IRB) in May 2012. The data collection protocol for this research was a three-step process. First, observational data was collected of the AEU process from two perspectives, the patient and the nurses who work in the unit. This allowed the researcher to view the process as it was happening from multiple views. Secondly, interviews were performed on ten different individuals, across different levels (doctors, nurses, nurse 
managers, administration), who work with in the AEU process. Thirdly, all documents pertaining to the AEU process were collected for comparison to what was actually happening in the AEU.

\subsubsection{Observations}

The patient observation occurred over three weeks in June of 2012, a time period in which the AEU staff said that they were below average in the number of patients present. Over the three weeks, each patient was followed from the time they entered the AEU until the time they left the AEU to be placed in a bed upstairs in the hospital. In total, 20 patients were observed over 14 days in which 110 hours were spent directly observing the AEU.

The nurse observations took place over a three week interval in January of 2013. Each nurse was followed multiple times over the course of three weeks for approximately four hours each day which allowed the researcher to view how the nurse handles multiple patients at the same time. Twenty-four hours of observation over eight days were performed observing the nurse with a total of $17 \mathrm{AEU}$ patient visits. The time period in which these observations took place was considered the hospital's peak season.

For both sets of observations, hand written notes were taken while an audio recording was made of the conversations that occurred for each patient. Upon completion of the observations, the hand written notes and audiotapes were transcribed anonymously to electronic format. The transcriptions were then entered into NVIVO@ (QSR International) for analysis. 


\subsubsection{Interviews}

The interviews with the personnel of the AEU took place in February of 2013. Since there were many steps to the AEU process, and not a single person saw every step, personnel from different groups were interviewed. In total, ten personnel were interviewed which account for four different groups; nurses/unit secretary (3), doctors (3), unit nurses mangers (3), and administration (1). Each interview lasted 30-75 minutes, was audiotaped, and was performed in a private area of the personnel's choice and scheduling. Upon completion of the interview, the audio tape was transcribed anonymously and inserted into NVIVOC (QSR International) for analysis.

As part of the interview process, each participant was asked to use a whiteboard to map out the process workflow of the AEU. Doing so allowed the researcher to understand how each person perceived the AEU workflow as it related to their job. Different process steps were provided on magnetic cards and the participants were asked to put them in the order that they see the AEU process happening. Blank cards were provided in case there was a particular step that had been left out that the participant felt was important. Upon completing the map of the workflow, each participant was asked to label any and all personnel that were associated with each step in the process. Also, each participant was asked to label any process steps they thought were a major source of delays to the process as a whole and any step they considered to be an inefficient process.

\subsubsection{Document Collection}

The collection of documents related to the AEU consisted of collecting any form used to collect patient data, any standing orders that are used in the AEU, and any written policies implemented in the AEU. These documents provided an understanding of what 
"should" happen in the AEU process. With the collection of the documents, an analysis could be performed to compare the actual events (observations), the perceived events (interviews), and the trained procedure (documents).

\subsection{Results}

The purpose of this research was to perform an assessment of the Admission Express Unit and provide recommendations for improvement to the administration of Kosair Children's Hospital. As described above, observational data was collected of the AEU process for two points of view and interviews were performed on the personnel who work with in the AEU. Upon completion of the data collection, a holistic process workflow was created of the AEU process with all associated personnel labeled, major delays identified, and inefficient process steps recognized. Figure 11 provides the process flow created from the observational data and interviews of the AEU staff. Table 5 provides a key for the abbreviations of personnel labeled and symbols in Figure 11. 
Table 5

Abbreviation Meanings and Key for Workflows

\begin{tabular}{|c|l|}
\hline$\underline{\text { Letter }}$ & \multicolumn{1}{|c|}{ People } \\
\hline A & Assistant Nurse Manager \\
\hline D & Referring Primary Care Physician \\
\hline E & EMS \\
\hline F & Family \\
\hline H & House Manager \\
\hline I & IV Team \\
\hline K & Respiratory Technician \\
\hline M & Doctor, Attending, Resident, Intern, Medical Student \\
\hline N & Nurse \\
\hline O & Other (Access Center, JFK, etc.) \\
\hline P & PCA \\
\hline Pt & Patient \\
\hline R & Registration \\
\hline S & Secretary \\
\hline T & Transport (Internal) \\
\hline V & Volunteer \\
\hline X & Radiology \\
\hline O & Delay \\
\hline O & Inefficient \\
\hline & \\
\hline
\end{tabular}




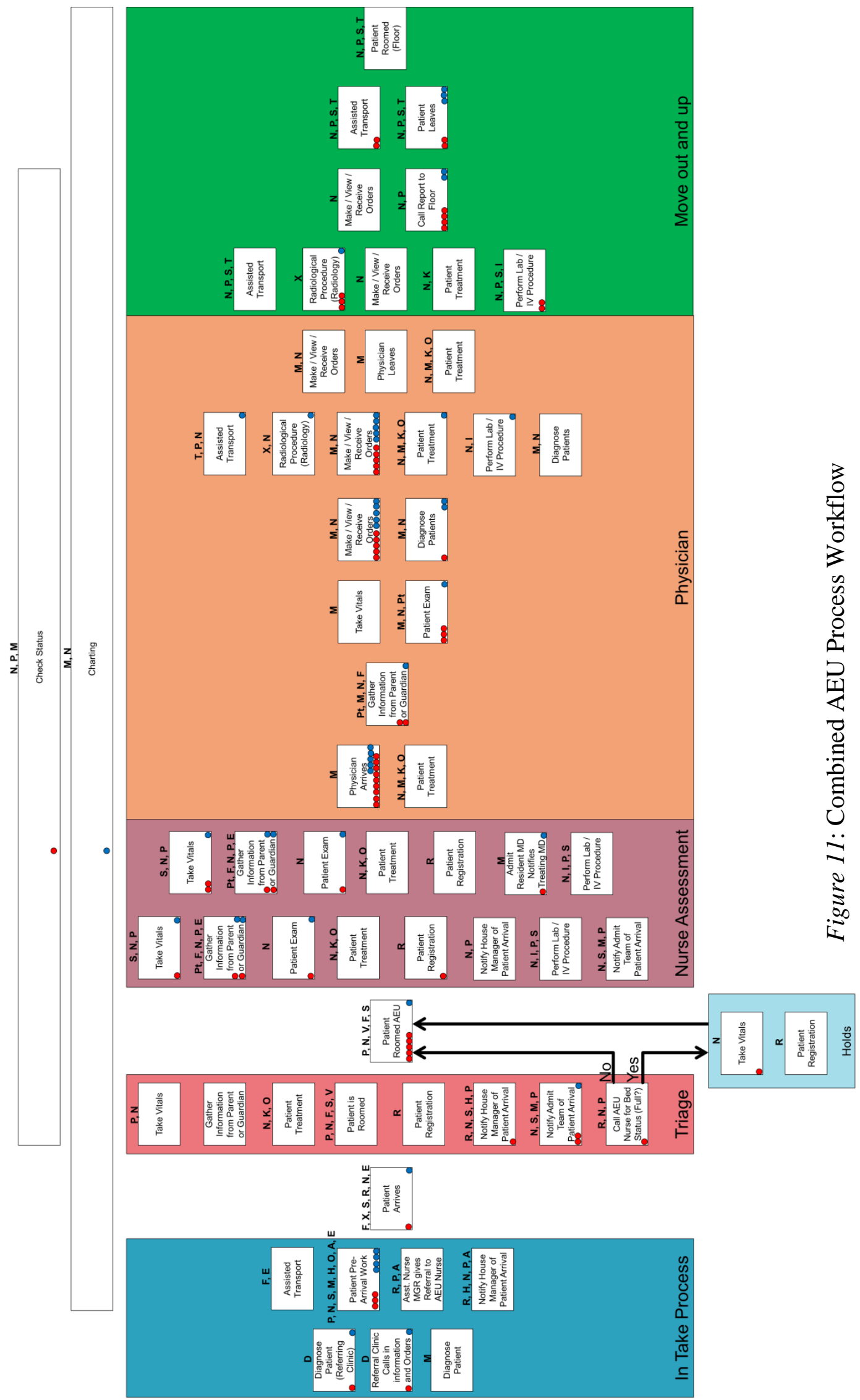


Figure 11 shows that there were five process steps that were identified as being problem areas by the personnel of the AEU:

1. Patient Pre-Arrival Work

2. Patient Roomed AEU

3. Physician Arrives

4. Make/View/Receive Orders

5. Call Report to Floor

Three of these processes are dependent on one another; Patient Pre-Arrival Work, Physician Arrives, and Make/View/Receive Orders. When the physician from an outside office calls in a patient, they are required to call three different people, the admitting doctor, the AEU nurse, and the house manager. If one person is not informed that a patient is coming to the AEU, then a delay is incurred because at some point the person who was not informed is finally made aware that there is a patient coming or already arrived and they are left scrambling to gather all the information they need to take care of the patient. Also, depending on a number of factors, the calling physician talk to someone from a call center that was not informed to collect the proper information; an Assistant Nurse Manager of the emergency department, or an aid/unit secretary; all of whom have a different set of information they collect and different forms they use to collect this information on.

While the Physician Arrives step was labeled as the step with the most delays and inefficiencies, it is mostly dependent on the Patient Pre-Arrival Work step working smoothly. If this step does not work smoothly, then the physicians are left trying to gather all the necessary information needed so that they are able to evaluate the patient. 
From the interviews and observations, it was also determined that the physicians who treat patients that enter through the AEU do not fully understand the goals of the AEU. According to the AEU policy, the physicians are supposed to see the patient no more than 15 minutes after the patient arrives in the AEU. In more than $50 \%$ of the cases observed, this did not happen. It was learned that many of physicians do not actually know that there is a time frame associated with visiting patients in the AEU. When the physicians were asked how they were trained on the policies and procedures of the AEU, it was stated by more than one person that they were taught everything they know about the AEU through a mentor. As a first year residents, they would round with older residents who would teach them what they needed to know on how to treat patients in the AEU.

The final process step in this group of three dependent steps is the Make/View/Receive Orders step. Again, this step is dependent on how well the previous step is executed. If the physician arrives in a timely fashion, then most of the time the orders are provided in a timely fashion. It is when the physician is delayed, whether it is because they are trying to rectify the Patient Pre-Arrival Work step or because they are being delayed elsewhere in the hospital, then the orders are perceived as being delayed. It was mostly the nurses and nurse managers that believed this process step to be a delay which is not surprising considering, at this point in the process of getting a patient through the AEU, they cannot do anything else until they are provided with orders from the physicians.

The Patient Roomed AEU had 50\% of the personnel stating that it was a process that was a significant delay. This can be explained with two different reasons. First, this process could tie into the above mentioned dependent set of processes in that if a patient 
arrives and the AEU personnel were unaware the patient was coming, there could be a delay in placing that patient into a room in the AEU. The more likely reason for this step being a delay is the capacity of the AEU at that time, which the AEU staff has no control over. The AEU has five patient rooms and one procedure room, which can be used as a patient room if needed. There are many occasions, especially during peak season, where all of the AEU rooms are being used and patients either have to be placed in emergency department rooms until they can be transferred over to the AEU or the patient will have to wait in the waiting room for a room to become available.

The final process step labeled by consensus as a delay and inefficient was the Call Report to Floor. While this process was labeled mostly as a major delay, it is out of the control of the AEU personnel. This delay stems from the hospital being at capacity and no beds being available. There were many instances where AEU patients were seen in the AEU in a timely fashion, but were required to wait many hours until they were able to be moved upstairs to a room. This delay does have an effect on the previous process step mentioned, the Patient Roomed AEU, because if a patient cannot be transferred upstairs when they are ready, then that patient is tying up a bed in the AEU that could be used for a new patient.

\subsection{Discussion}

With the identification of the problem areas of the AEU process, the question remains, "How do we potentially rectify these problem areas to improve the process of the AEU?" Hence, Table 6 provides a ranked list of the failures observed in the AEU and the reason(s) why it failed. This list was ranked based on how problematic the failure is to the overall AEU process as seen throughout this study. Also provided is evidence 
from the observations and/or interviews as to some reasons why it is a problem and

potentially why it fails. Finally, potential fixes are provided.

Table 6

Failures in the AEU and Potential Fixes

\begin{tabular}{|c|c|c|c|c|}
\hline Rank & Failure & Why it Failed & Potential Fix & $\begin{array}{c}\text { Observational or } \\
\text { Interview Supporting } \\
\text { Data }\end{array}$ \\
\hline 1 & $\begin{array}{l}\text { The Call- } \\
\text { in/Pre- } \\
\text { Arrival } \\
\text { Process }\end{array}$ & $\begin{array}{l}\text { - Multiple KCH } \\
\text { people needed to } \\
\text { be contacted } \\
\text { - Unfamiliarity of } \\
\text { some outside } \\
\text { physicians with } \\
\text { KCH system } \\
\text { - KCH has no } \\
\text { standardized data } \\
\text { collection between } \\
\text { units/people } \\
\text { - "Unstable" } \\
\text { patients are sent to } \\
\text { the AEU }\end{array}$ & $\begin{array}{l}\text { - One point of } \\
\text { contact for } \\
\text { physicians calling } \\
\text { - Single data } \\
\text { collection form to } \\
\text { confirm status, } \\
\text { diagnosis, and } \\
\text { basic information } \\
\text { - Strict definition of } \\
\text { the AEU type of } \\
\text { patient }\end{array}$ & $\begin{array}{l}\text { - "One of the most } \\
\text { frustrating things is the } \\
\text { beginning" } \\
\text { - "Either the patient } \\
\text { arrives without anyone } \\
\text { being aware of them } \\
\text { coming in the first } \\
\text { place" } \\
\text { "We don't have a set } \\
\text { pre-arrival intake } \\
\text { form" } \\
\text { - } 3 \text { out of } 10 \text { said that if } \\
\text { they would fix one } \\
\text { thing this step would } \\
\text { be it }\end{array}$ \\
\hline 2 & $\begin{array}{l}\text { The } \\
\text { Physician } \\
\text { arrival to the } \\
\text { AEU in } 15 \\
\text { minutes }\end{array}$ & $\begin{array}{l}\text { - Failure of the } \\
\text { Call-In Process } \\
\text { causes Physician } \\
\text { to be unaware of } \\
\text { patient, lack } \\
\text { information, etc. } \\
\text { - Physicians } \\
\text { unaware of AEU } \\
\text { policies } \\
\text { - Physicians } \\
\text { providing care to } \\
\text { non-AEU patients }\end{array}$ & $\begin{array}{l}\text { - Address the Call- } \\
\text { in/Pre-Arrival } \\
\text { Process problem } \\
\text { - Provide training to } \\
\text { the new residents } \\
\text { when they enter } \\
\text { into KCH each } \\
\text { year } \\
\text { - Have a permanent } \\
\text { physician or nurse } \\
\text { practitioner in the } \\
\text { AEU }\end{array}$ & $\begin{array}{l}\text { - } 9 \text { out of } 10 \text { personnel } \\
\text { label process as a delay } \\
\text { - } 5 \text { out of } 10 \text { personnel } \\
\text { label process as } \\
\text { inefficient } \\
\text { o } 3 \text { of the } 5 \text { rank } \\
\text { process as \#1 } \\
\text { inefficient process } \\
\text { - "I've known where the } \\
\text { nurse calls and says, } \\
\text { you know, this } \\
\text { patient's been here a } \\
\text { really long time and } \\
\text { they're asking for you, } \\
\text { and I didn't know they } \\
\text { were there." } \\
\text { "I think the goal is } 15 \\
\text { minutes and we wait } \\
30 \text { minutes to } 2 \text { hours } \\
\text { most of the time." }\end{array}$ \\
\hline
\end{tabular}




\begin{tabular}{|c|c|c|c|c|}
\hline 3 & $\begin{array}{l}\text { Physician } \\
\text { providing } \\
\text { orders to } \\
\text { nurse }\end{array}$ & $\begin{array}{l}\text { - Dependent of } \\
\text { Call-in/Pre- } \\
\text { Arrival and } \\
\text { Physician Arrival } \\
\text { happening in a } \\
\text { timely fashion } \\
\text { - Unnecessary steps } \\
\text { being performed, } \\
\text { i.e. consultations } \\
\text { and treatments } \\
\text { - Physicians } \\
\text { teaching residents }\end{array}$ & $\begin{array}{l}\text { - Produce additional } \\
\text { standing orders for } \\
\text { particular } \\
\text { diagnosis } \\
\text { - Do not allow } \\
\text { consultations to } \\
\text { impact flow in the } \\
\text { AEU }\end{array}$ & $\begin{array}{l}\text { - "They [admitting } \\
\text { doctor] may send other } \\
\text { people to collect the } \\
\text { data, and then they're } \\
\text { going to come or } \\
\text { they're going to review } \\
\text { it by phone" } \\
\text { - "So we might get the } \\
\text { orders in a little bit } \\
\text { late." } \\
\text { - "Well I think that } \\
\text { waiting a long time for } \\
\text { the doctors to write } \\
\text { orders is inefficient." }\end{array}$ \\
\hline 4 & $\begin{array}{l}\text { Admitting } \\
\text { Doctor with } \\
\text { pager }\end{array}$ & $\begin{array}{l}\text { - Has to delegate } \\
\text { other physicians } \\
\text { to round on } \\
\text { patients when } \\
\text { they arrive } \\
\text { - Constantly being } \\
\text { interrupted while } \\
\text { visiting patient }\end{array}$ & $\begin{array}{l}\text { - Have physician } \\
\text { with the pager not } \\
\text { round on patients } \\
\text { for that day } \\
\text { - Allow them to } \\
\text { receive call and } \\
\text { delegate patients } \\
\text { to the other } \\
\text { resident teams } \\
\text { - Nurse Practitioner } \\
\text { or physician } \\
\text { assigned to the } \\
\text { AEU }\end{array}$ & $\begin{array}{l}\text { - "Sometimes there } \\
\text { might be a delay here, } \\
\text { depending on how } \\
\text { many times they are } \\
\text { beeped." } \\
\text { " "Get that dang gone } \\
\text { admission pager away } \\
\text { from one of the admit } \\
\text { team." } \\
\text { "I am busy taking } \\
\text { phone calls as the } \\
\text { admit resident" }\end{array}$ \\
\hline 5 & $\begin{array}{l}\text { Using AEU } \\
\text { for teaching } \\
\text { purposes }\end{array}$ & $\begin{array}{l}\text { - Rooms not big } \\
\text { enough } \\
\text { (congestion) } \\
\text { - "Express" unit } \\
\text { patient admission } \\
\text { and teaching } \\
\text { students are } \\
\text { conflicting goals }\end{array}$ & $\begin{array}{l}\text { - Care should be } \\
\text { provided only by } \\
\text { senior residents }\end{array}$ & $\begin{array}{l}\text { - "A lot of times when } \\
\text { they examine the } \\
\text { patient, they bring } \\
\text { students with them and } \\
\text { they are not really } \\
\text { supposed to" } \\
\text { "They may send other } \\
\text { people to collect the } \\
\text { data, and then they're } \\
\text { going to come or } \\
\text { they're going to review } \\
\text { it by phone" }\end{array}$ \\
\hline
\end{tabular}




\begin{tabular}{|c|c|c|c|c|}
\hline 6 & $\begin{array}{l}\text { Unnecessary } \\
\text { steps being } \\
\text { performed in } \\
\text { AEU }\end{array}$ & $\begin{array}{l}\text { - Beginning } \\
\text { treatment of } \\
\text { patient in the } \\
\text { AEU, which falls } \\
\text { outside scope of } \\
\text { the AEU } \\
\text { - Consultations } \\
\text { with specialists }\end{array}$ & $\begin{array}{l}\text { - Specialist should } \\
\text { wait until the } \\
\text { patient is in their } \\
\text { room upstairs until } \\
\text { they visit } \\
\text { - Treatments that } \\
\text { are not time } \\
\text { sensitive with } \\
\text { respect to outcome } \\
\text { should be given } \\
\text { upstairs in patient } \\
\text { room }\end{array}$ & $\begin{array}{l}\text { - } 2 \text { instances of } \\
\text { consultations resulted } \\
\text { in doctor visiting } \\
\text { patient in AEU for } 57 \\
\text { min. and } 37 \text { min. each } \\
\text { "They often order } \\
\text { treatments that really } \\
\text { do not fall under our } \\
\text { guidelines but want it } \\
\text { done which increases } \\
\text { their time" }\end{array}$ \\
\hline 7 & $\begin{array}{l}\text { Looking for } \\
\text { or retrieving } \\
\text { supplies }\end{array}$ & $\begin{array}{l}\text { Having to stop a } \\
\text { procedure to } \\
\text { retrieve an item } \\
\text { Having to travel } \\
\text { out of the } \\
\text { department to get } \\
\text { a supply }\end{array}$ & $\begin{array}{l}\text { Evaluate the } \\
\text { economic order } \\
\text { quantity (EOQ) to } \\
\text { help ensure the } \\
\text { necessary supplies } \\
\text { are in the AEU }\end{array}$ & $\begin{array}{l}\text { - } 14 \text { instances of a nurse } \\
\text { looking for a supply } \\
\text { - "Nurse has Aid go get } \\
\text { a new and bigger } \\
\text { catheter." }\end{array}$ \\
\hline
\end{tabular}

By far, the most important step of the AEU process is the Call-in/Pre-Arrival

Process as so many other processes and the fluidity of the whole process relies on this step working properly. Multiple personnel stated that this process was the biggest issue with the AEU and fixing the process would help to eliminate a lot of the issues associated with the long amount of time required to get through the AEU process. The issue with fixing this step is that there are many factors that can contribute to Call-in/Pre-Arrival Process not working smoothly, such as, multiple individuals needed to be contacted, standardizing the data collected, and ensuring that only stable patients are sent to the AEU. Each of these factors would need to be addressed to potentially expedite and make this process more efficient.

To address the factor of multiple individuals needing to be contacted, the obvious resolution is to have one person as the AEU contact person physicians to call. The question is who should this contact person be? At one point, $\mathrm{KCH}$ had tried to implement this approach, and created the Access Center. According to AEU personnel, 
the issue with the Access Center was that the person who was receiving the calls was not qualified to do so. This person did not have the knowledge needed to ask the right questions to gain a true understanding of the condition of the patient. So it is recommended that a person be appointed as the contact person for the AEU who has:

- The knowledge of the type of patient that should and should not be in the AEU

- Have the authority to accept a patient to the AEU or refer a patient the emergency department based on the patient condition

- Have the knowledge to ask the appropriate questions needed for the personnel of the AEU to be able to be prepared to assess the patient upon arrival.

The second factor that needs to be addressed is standardizing the data collected. To meet this goal, one data sheet needs to be created that all personnel throughout the hospital use, even if there are multiple points of contact that primary care physicians might have to call. It has been suggested that primary care phsicians are not satisified with communication at transition points because communication is not provided in a timely manner, omits essential information, or contains ambiguities that put patients at risk (Johnson, et al., 2012; Kripalani, LeFevre, Phillips, Williams, Basaviah, \& Baker, 2007). This can be seen in $\mathrm{KCH}$ and shows the need for the AEU to have a standardized data collection form that omits the ambiguties and contains questions to receive the essential information quickly.

Addressing the above mentioned problem of standardizing data collect along with addressing the issue of ensuring only stable patients be admitted through the AEU can be rectified with a tool. The Pedatric Early Warning Score (PEWS) is an early warning 
system that is used to assess and identify patients who were deteriorating (Duncan, Hutchison, \& Parshuram, 2006; Tucker, Brewer, Baker, Demeritt, \& Vossmeyer, 2009). The PEWS assesses the child's behavior, cardiovascular condition, and respiratory condition and assigns points appropriately. The sum of the assigned points provides the care providers with a recommended time interval that the child should be reassessed. It is the recommendation that an adapted version of the PEWS be created for use in the admission process in $\mathrm{KCH}$. The three areas of assessment that are currently used in the PEWS is information that is already collected during the admission process. If an adaptation could be created that was one form that anyone collecting the patient informaiton could use, which collects all of the necessary information needed for admission while providing the proper information needed to determine the stability of patients, then there is potential for the Call-in/Pre-Arrival process to work more efficiently. Personnel of $\mathrm{KCH}$ would have to work together to ensure all necessary areas for assessment were included but at the same time, determine a way to include the necessary information in a consise form.

Another recommendation for improvement of the AEU process is to provide training to the new residents each year which would inform them of the purpose, policies, and procedures of the AEU. Imporant information that should be included in this training, which would potentially help improve the arrival time of the physicians, would be the requirement to be in the AEU 15 minutes after the arrival of the patient. According to a worker in the AEU, "A lot of them [physicians] don't know that they are supposed to be here in 15 minutes. They will say, well I didn't know that or they are just tied up with other kids in other areas and they can't make it." Information about the type 
of patients that should be and should not be accepted to the AEU would also be an important topic to discuss. AEU personnel stated, "They are not informed that if they get a call on a kid that sounds bad, that they can refuse it and refer it to the ER. A lot of them are like; well I didn't know I could do that. I think a lot of that is just poor education on whoever is supposed to inform them of what the Admit Express is." It is obvious to the staff of the AEU that if all employees were more informed, things could potentially run smoother within the unit.

A recommendation that could potentially improve the overall process of the AEU would be to not allow the admit resident who has the admit pager for that day to round on patients. It was stated by more than one person during the interviews that if the resident who was carrying the admit pager for that day was rounding on a patient in the AEU, then there were more delays with that patient because the resident was required to return many pages. However, this recommendation would require major change to the operating procedures of the physicians of $\mathrm{KCH}$. An alternative solution would be to develop more standing orders that the physicians could activate by verbal orders to the nurse. Currently, the AEU has standing orders for some illnesses such as Pyloric Stenosis and Abscesses. However, the emergency department has standing orders for additional illnesses that the AEU does not. The addition of more standing orders for various illnesses could potentially increase the efficiency of the AEU and help get children with these illnesses admitted more rapidly.

The final recommendation is concerned with the Triage Process. Unique to $\mathrm{KCH}$ is the AEU. It is unique in that a triage process is not needed for patients going through the AEU if the Call-in/Pre-Arrival step works properly, which is the current standpoint of 
$\mathrm{KCH}$. However, a triage process was observed, whether it is recognized and documented is a different issue. This triage process normally consisted of the nurse determining the stability of the patient and whether there was need for the patient to be transferred to the ED. Knowing and seeing that a triage process is being performed begs the question as to whether this process is needed. Hence, the recommendation is that $\mathrm{KCH}$ determine if this process is needed. If it is not needed, would creating a form, as mentioned above, help to eliminate the need for patients to be triaged when they arrive to the AEU. If the process is needed, is there a way to shorten the process currently used by the ED or is there a need for a full triage process to be developed specifically for the AEU.

\subsubsection{Potential Limitations}

A potential limitation to this research study is the time of the year that both the patient observations and the nurse observations/interviews took place. While it was beneficial for the researcher to see the AEU at its two extremes, an unusual slow time for the AEU for the patient observations in the summer and peak season for the nurse observations in the winter, it would also have been beneficial to have observed the AEU in the spring and fall to ensure that all time periods were observed.

Another limitation to this study was the inability to observe the Call-in/PreArrival process from the physician's point of view. Due to the other responsibilities that the physicians had to other areas of the hospital, it was inefficient to observe the physicians other than the times they were present in the AEU. However, the inability of the researchers to view this particular process required the researcher to rely solely on the interview process to gain the knowledge and information, from the physicians, concerning the process. 


\subsection{Conclusions}

The purpose of this study was to evaluate the current AEU process to determine areas for potential improvement. It was discovered that the Call-in/Pre-Arrival process was the area with the greatest need for improvement. With improvements to the area, many of the issues observed by the researcher and mentioned by the staff of the AEU could potentially be resolved. Most of the failures mentioned in this research are the result of communication. Whether the failure has to do with the Call-in/Pre-Arrival process to the physician not arriving to the AEU in 15 minutes or the resident having the admit pager while rounding on patients in the AEU, each failure can trace the root of its problem back to communication or lack thereof.

There were many areas identified for potential improvement and recommendations to meet those needs discussed within this research. A list of the nine most important "do's" and "don'ts" is provided for any hospital that either wants to improve or wants to introduce a rapid admission unit.

1. Do: Identify one point of contact for physicians to call to admit patients

2. Do Not: Allow a resident rounding on patients be the admit resident for that day

3. Do: Have one form that collects all necessary information while identifying the stability of the patient

4. Do Not: Use the rapid admission unit as a teaching area for the new residents

5. Do: Train the new residents each year in the policies and procedures of the rapid admission unit

6. Do Not: Perform consultations and treatments with patients in the rapid admissions unit 
7. Do: Identify the purpose of the rapid admission unit and educate everyone associated with the unit to the goals, policies, and procedures

8. Do Not: Utilize the resources dedicated to the rapid admission unit for other purposes unless and emergency exists, i.e. rooms, beds, supplies, etcetera

9. Do: Make the AEU a 24 hour unit

\subsubsection{Future Work}

With identifying the complete workflow of the AEU and mapping it out step-bystep, additional potential areas of research presented itself. A potential benefit to $\mathrm{KCH}$ would be the creation of a Discrete Event Simulation (DES) model. With the process already mapped out in detail and easily being able to capture the time to complete each process through the observational data, a computer simulation could be made that would provide additional support for where potential problems are with in the process. Also, an advantage of a DES model is the ability to test changes to the system without actually implementing them in real life to determine what effect a change like that would have on the overall system. Ultimately, this could potentially save $\mathrm{KCH}$ time and money be being able to test a multitude of different scenarios at little cost. 


\section{CHAPTER 6: CONCLUSIONS AND FUTURE RESEARCH}

The purpose of this research was to evaluate the Admission Express Unit of Kosair Children's Hospital to determine potential areas for improvement to the process. The amount of time for patients to be seen in the AEU had increased over the last few years. Also, the purpose of the AEU had morphed from its original intentions of a unit that collects patient information, allow the physicians to perform an evaluation, and perform any IVs, labs, or x-rays needed to a unit that does all of the previously mentioned as well as begin treatment and consultations. Concern was raised when several adverse events, including one event requiring resuscitation, occurred that were attributed in part to delays in the admission process.

Several evaluation techniques were used in the analysis of the AEU. First, direct observations were performed of the unit from both the patient's point of view and nurse's point of view. Secondly, one-on-one interviews were performed with 10 different personnel who work in or with the AEU. From this analysis, several observations were made:

- $\quad 37 \%$ of the deviated events or failures, which resulted in delays, were found in the Nurse Assessment

- The highest average time to recovery, 32 minutes, was in the Intake Process 
- Based on the interviews, 5 areas were identified as problem areas for the AEU: 1) Patient Pre-Arrival Work, 2) Patient Roomed AEU, 3) Physician Arrives, 4) Make/View/Receive Orders, and 5) Call Report to Floor

- 3 of the problem areas are dependent on one another: Patient Pre-Arrival Work, Physicians Arrives, and Make/View/Receive Orders

- The Call-in/Patient Pre-Arrival Process is the most important process of the AEU as the remaining processes rely on this step working properly With these observations made, a list of recommendations was developed that could potential assist in improving the AEU process:

- Identify one point of contact for physicians to call to admit patients

- Have the contact person use one form that collects all necessary information while identifying the stability of the patient

- Train new residents each year in the policies and procedures of the AEU

- Perform consultations and treatments with patients once they have reached their room in the hospital

- Refrain from using the AEU as a teach area for the new residents

\subsection{Future Work}

As it has been already stated, there is a direct relation between timeliness of admission to treatment effectiveness to patient outcomes. Delays in healthcare processes are becoming more of a problem in hospitals today (Lane, Monefeldt, \& Rosenhead, 2000; Horwitz \& Bradley, 2009; Stone, Boehme, Mundorff, Maloney, \& Sriastava, 2010), which prevent proper treatment to patients. Future research needs to extend this study of an admission unit to other admission unit for comparison. The unique feature of 
the $\mathrm{AEU}$ is that it does not require patients to go through the emergency room to be admitted and, if the unit is functioning properly, does not require a triage of the patient to be admitted. A comparison of the AEU to other admission units in other hospitals will allow researchers to determine if one method of admission is superior to another. Doing so will help meet the goal of a decreased time of admission which can be directly attributed to the goals of healthcare and the best interest of patients, i.e. patient safety. 


\section{REFERENCES}

Ahluwalia, J., \& Marriott, L. (2005). Critical incident reporting systems. Seminars in Fetal \& Neonatal Medicine, 31-37.

Baker, D. W., Lindquist, L., Liss, D., \& Noskin, G. A. (2010). Results of the Medications at Transitions and Clinical Handoffs (MATCH) study: an analysis of medication reconciliation errors and risk factors at hospital admission. Journal of General Internal Medicine, 441-447.

Ben-Tovin, D. I., Dougherty, M. L., O'Connell, T. J., \& McGrath, K. M. (2008, March 17). Patient journeys: the process of clinical redesign. Medical Journal of Australia, 188(6), S14-S17.

Boan, D., Nadzam, D., \& Clapp Jr., J. R. (2012). The impact of variance in perception of the organization on capacity to improve in hospital work groups. Group Dynamics: Theory, Research, and Practice, 16(3), 206-217.

Boston-Fleishhauer, C. (2008). Enhancing healthcare process design with human factors engineering and reliability science, part 1. The Journal of Nursing Administration, $38,27-32$.

Carayon, P., Hundt, A., Karsh, B., Gurses, A., Alvarado, C., Smith, M., et al. (2006). Work system design for patient safety: the SEIPS Model. Qual Safety Healthcare, $50-58$. 
Cornish, P. L., Knowles, S. R., Marchesano, R., Tam, V., Shadowist, S., Juurlink, D., et al. (2005). Unintended medication discrepancies at the time of hospital admission. JAMA Internal Medicine, 165(4), 424-429.

Creswell, J. W. (2009). Research Design: Qualitative, Quantitative, and Mixed Methods Approaches. Thousand Oaks, CA: Sage Publications.

de Bucourt, M., Busse, R., Guttler, F., Reinhold, T., Vollnberg, B., Kentenich, M., et al. (2012). Process mapping of PTA and stent placement in a university hospital interventional radiology department. Insights Mapping, 3, 329-336.

Duncan, H., Hutchison, J., \& Parshuram, C. (2006). The Pediatric Early Warning System score: a severity of illness score to predict urgent medical need in hospitalized children. Journal of critical care, 21(3), 271-278.

Flanagan, D., Ellis, J., Baggott, A., Grimsehl, K., \& English, P. (2010). Diabetes management of elective hospital admissions. Diabetic Medicine, 27(11), 12891294.

Hoonakker, P. L., Cartmill, R. S., Carayon, P., \& Walker, J. M. (2011). Development and psychometric qualities of the SEIPS Survey to evaluate CPOE/HER implementation in ICUs. Int J Healthc Inf Syst Inform, 51-69.

Horwitz, L. I., \& Bradley, E. (2009). Percentage of US emergency department patients seen within the recommended triage time. Archives of Internal Medicine, 169(20), $1857-1865$. 
Huang, Q., Thind, A., Dreyer, J. F., \& Zaric, G. S. (2010). The impact of delays to admission from the emergency department on inpatient outcomes. $B M C$ emergency medicine, 1-6.

Hysong, S. J., Sawhney, M. K., Wilson, L., Sittig, D. F., Esquivel, A., Watford, M., et al. (2009). Improving outpatient safety through effective electronic communication: A study protocol. Implementation Science, 4, 62.

Institute of Medicine. (1999). To err is human: Building a safer health system. Washington, DC: National Academies Press.

Johnson, J. K., Farnan, J. M., Barach, P., Hesselink, G., Wollersheim, H., Pijnenborg, L., et al. (2012). Searching for the missing pieces between the hospital and primary care: mapping the patient process during care transitions. British Medical Journal of Quality and Safety, 1-9.

Joint Commission on Accreditation of Healthcare Organization and American Society for Healthcare Risk Management. (2004). Accrediitation Issues for Risk Managers.

Kafetz, K. (2010). How effective are acute geriatric wards at admitting geriatric patients? Royal College of Physicians, 420-421.

Keenan, S. P., Doig, G. S., Martin, C. M., Inman, K. J., \& Sibbald, W. J. (1997). Assessing the efficiency of the admission process to a critical care unit: does the literature allow the use of benchmarking? Intensice Care Med, 574-580. 
King, D. L., Ben-Tovim, D. I., \& Bassham, J. (2006). Redesigning emergency department patient flows: Application of Lean thinking to health care. Emergency Medicine Australasia, 18, 391-397.

Kripalani, S., LeFevre, F., Phillips, C. O., Williams, M. V., Basaviah, P., \& Baker, D. W. (2007). Deficits in communication and information transfer between hospitalbased and primary care physicians. JAMA: The Journal of the American Medical Association, 297(8), 831-841.

Lane, D. C., Monefeldt, C., \& Rosenhead, J. B. (2000, May). Looking in the wrong place for healthcare improvements: a system dynamics study of an accident and emergency department. The Journal of the Operational Research Society, 51(5), $518-531$.

Latino, R. J. (2004). Optimizing FMEA and RCA efforts in heatlh care. ASHRM Journal, $24,21-28$.

Marshall, C., \& Rossman, G. B. (2006). Designing Qualitative Research (4th ed. ed.). Thousand Oaks, CA: Sage Publications.

Mazzocato, P., Holden, R. J., Brommels, M., Aronsson, H., Backman, U., Elg, M., et al. (2012). How does lean work in emergency care? A case study of a lean-inspired intervention at the Astrid Lindgren Children's hosptial, Stockhold, Sweden. BMC Health Services Research, 12, 28.

Rhudy, L., Hollan, D. E., \& Bowles, K. H. (2010). Illuminating hospital discharge planning: staff nurse decision making. Applied Nursing Research, 198-206. 
Simon, T., Berry, J., Feudtner, C., Stone, B. L., Sheng, X., Bratton, S. L., et al. (2010). Children with complex chronic conditions in inpatient hospital setting in the United States. Pediatrics, 126(4), 647-655.

Stone, B. L., Boehme, S., Mundorff, M. B., Maloney, C. G., \& Sriastava, R. (2010). Hospital admission medication reconciliation in medically complex children: an observational study. Archives of Disease in Childhood, 95, 250-255.

Subbe, C., Jishi, F., \& Hibbs, R. (2010). The Simple Clinical Score: a tool for benchmarking of emergency admissions in acute internal medicine. Clinical Medicine, 10(4), 352-357.

Trebble, T. M., Hansi, N., Hydes, T., Smith, M. A., \& Baker, M. (2010). Process mapping the patient journey through health care: an introduction. BMJ, 341, 394397.

Tucker, K. M., Brewer, T. L., Baker, R. B., Demeritt, B., \& Vossmeyer, M. T. (2009). Prospective evaluation of a pediatric inpatient early warning scoring system. Journal for Specialists in Pediatric Nursing, 14(2), 79-85.

Unroe, K. T., Pfeiffenberger, T., Riegelhaupt, S., Jastrzembski, J., Lokhnygina, Y., \& Colón-Emeric, C. (2010). Inpatient medication reconciliation at admission and discharge: A retrospective cohort study of age and other risk factors for medication discrepancies. The American Journal of Geriatric Pharmacotherapy, 115. 
Walker, B., \& Haslett, T. (2001). System dynamics and action research in aged care. Australian Health Review, 24(1), 183-191.

Weigl, M., Muller, A., Vincent, C., Angerer, P., \& Sevdalis. (2011). The association of workflow interruptions and hospital doctors' workload: a prospective observational study. BMJ Quality and Safety, 399-407.

Weigl, M., Muller, A., Zupanc, A., Glaser, J., \& Angerer, P. (2011, January). Hospital doctors' workflow interruptions and activities: an observation study. BMJ Quality and Safety, 491-497.

Wetterneck, T. B., \& Holman, G. T. (2011). Use of tandem observations in ambulatory primary care to evaluate physician-nurse teamwork. International Conference on Healthcare Systems Ergonomics and Patient Safety (HEPS) (pp. 163-166). Oviedo, Spain: Taylor \& Francis Group.

Wreathall, J., \& Nemeth, C. (2003). Assessing Risk: the Role of Probabilistic Risk Assessment (PRA) in Patient Safety Improvement. Qual Safety Healthcare, 13, 206-212. 


\section{APPENDIX A: DEVIATED EVENTS OR FAILURES OF THE AEU}

Table 7

Deviated Events and/or Failures of the AEU

\begin{tabular}{|c|c|c|c|c|c|}
\hline $\begin{array}{l}\text { Process } \\
\text { Step }\end{array}$ & $\begin{array}{c}\text { Type } \\
\text { of Obs. }\end{array}$ & Day & Patient & $\begin{array}{c}\text { Time to } \\
\text { Recovery (min) }\end{array}$ & $\begin{array}{l}\text { Type of Deviated } \\
\text { Event/Failure }\end{array}$ \\
\hline \multirow{4}{*}{$\begin{array}{l}\text { Intake } \\
\text { Process }\end{array}$} & Nurse & 5 & PT1 & 33 & Communication \\
\hline & Nurse & 8 & PT6 & 16 & Communication \\
\hline & Patient & 8 & PT1 & 53 & Communication \\
\hline & Patient & 12 & PT2 & 26 & Communication \\
\hline Triage & Nurse & 2 & PT2 & 5 & Person Does Not Show Up \\
\hline \multirow{21}{*}{$\begin{array}{c}\text { Nurse } \\
\text { Assessment }\end{array}$} & Nurse & 1 & PT3 & 25 & Communication \\
\hline & Nurse & 1 & PT3 & 3 & Family/Patient needs \\
\hline & Nurse & 2 & PT1 & 1 & Equipment/Supplies \\
\hline & Nurse & 2 & PT1 & 1 & Communication \\
\hline & Nurse & 2 & PT2 & 2 & Person Does Not Show Up \\
\hline & Nurse & 2 & PT2 & 1 & Equipment/Supplies \\
\hline & Nurse & 3 & PT2 & 3 & Equipment/Supplies \\
\hline & Nurse & 3 & PT4 & 1 & Equipment/Supplies \\
\hline & Nurse & 3 & PT4 & 29 & Equipment/Supplies \\
\hline & Nurse & 4 & PT2 & 4 & Equipment/Supplies \\
\hline & Nurse & 4 & PT4 & 1 & Communication \\
\hline & Nurse & 6 & PT2 & 1 & Communication \\
\hline & Nurse & 6 & PT2 & 1 & Communication \\
\hline & Nurse & 8 & PT2 & 10 & Equipment/Supplies \\
\hline & Nurse & 8 & PT4 & 2 & Equipment/Supplies \\
\hline & Patient & 1 & PT2 & 1 & Equipment/Supplies \\
\hline & Patient & 3 & PT1 & 2 & Other \\
\hline & Patient & 3 & PT3 & 7 & Communication \\
\hline & Patient & 3 & PT3 & 1 & Communication \\
\hline & Patient & 4 & PT1 & 1 & Communication \\
\hline & Patient & 4 & PT2 & 1 & Communication \\
\hline
\end{tabular}




\begin{tabular}{|c|c|c|c|c|c|}
\hline & Patient & 7 & PT1 & 18 & Other \\
\hline & Patient & 7 & PT2 & 20 & Person Does Not Show Up \\
\hline & Patient & 8 & PT1 & 1 & Communication \\
\hline & Patient & 11 & PT1 & 13 & Person Does Not Show Up \\
\hline \multirow{16}{*}{ Physician } & Nurse & 2 & PT1 & $93+$ & Person Does Not Show Up \\
\hline & Nurse & 3 & PT1 & 2 & Communication \\
\hline & Nurse & 6 & PT2 & 11 & Communication \\
\hline & Nurse & 8 & PT2 & 7 & Communication \\
\hline & Nurse & 8 & PT4 & 1 & Communication \\
\hline & Patient & 1 & PT1 & 2 & Communication \\
\hline & Patient & 3 & PT2 & 12 & Communication \\
\hline & Patient & 4 & PT1 & 57 & Consultation \\
\hline & Patient & 4 & PT2 & 2 & Communication \\
\hline & Patient & 5 & PT1 & $12+$ hours & Communication \\
\hline & Patient & 5 & PT1 & 21 & Communication \\
\hline & Patient & 6 & PT1 & 7 & Communication \\
\hline & Patient & 6 & PT2 & 48 & Communication \\
\hline & Patient & 7 & PT1 & 6 & Other \\
\hline & Patient & 9 & PT1 & 38 & Communication \\
\hline & Patient & 11 & PT1 & 33 & Consultation \\
\hline \multirow{17}{*}{$\begin{array}{c}\text { Move Out } \\
\text { and Up }\end{array}$} & Nurse & 2 & Hold & 1 & Communication \\
\hline & Nurse & 3 & PT3 & 2 & Communication \\
\hline & Nurse & 3 & PT3 & 1 & Communication \\
\hline & Nurse & 3 & PT3 & 3 & Equipment/Supplies \\
\hline & Nurse & 5 & PT1 & 3 & Equipment/Supplies \\
\hline & Nurse & 5 & PT1 & 15 & House Delay/Full \\
\hline & Nurse & 6 & PT2 & $36+$ & House Delay/Full \\
\hline & Nurse & 8 & PT1 & 245 & House Delay/Full \\
\hline & Patient & 5 & PT1 & 10 & Communication \\
\hline & Patient & 5 & PT1 & 74 & Communication \\
\hline & Patient & 6 & PT1 & 22 & Communication \\
\hline & Patient & 6 & PT1 & 80 & House Delay/Full \\
\hline & Patient & 7 & PT2 & 5 & Equipment/Supplies \\
\hline & Patient & 7 & PT2 & 12 & Other \\
\hline & Patient & 9 & PT1 & 3 & Communication \\
\hline & Patient & 12 & PT2 & 3 & Equipment/Supplies \\
\hline & Patient & 12 & PT2 & 18 & Other \\
\hline
\end{tabular}




\begin{tabular}{|c|c|c|c|c|c|}
\multirow{4}{*}{} & Nurse & 1 & N/A & 2 & Equipment/Supplies \\
\cline { 2 - 6 } & Nurse & 2 & N/A & 2 & Equipment/Supplies \\
\cline { 2 - 6 } & Nurse & 2 & N/A & 1 & Equipment/Supplies \\
\cline { 2 - 6 } & Nurse & 2 & N/A & 2 & Equipment/Supplies \\
\cline { 2 - 6 } & Nurse & 4 & N/A & 2 & Family/Patient needs \\
\cline { 2 - 6 } & Nurse & 6 & N/A & 1 & Equipment/Supplies \\
\cline { 2 - 6 } & Nurse & 6 & N/A & 8 & Equipment/Supplies \\
\cline { 2 - 6 } & Nurse & 8 & N/A & 10 & Equipment/Supplies \\
\hline
\end{tabular}




\section{APPENDIX B: ANOVA}

\section{General Linear Model: BOXC1 versus Process Step}

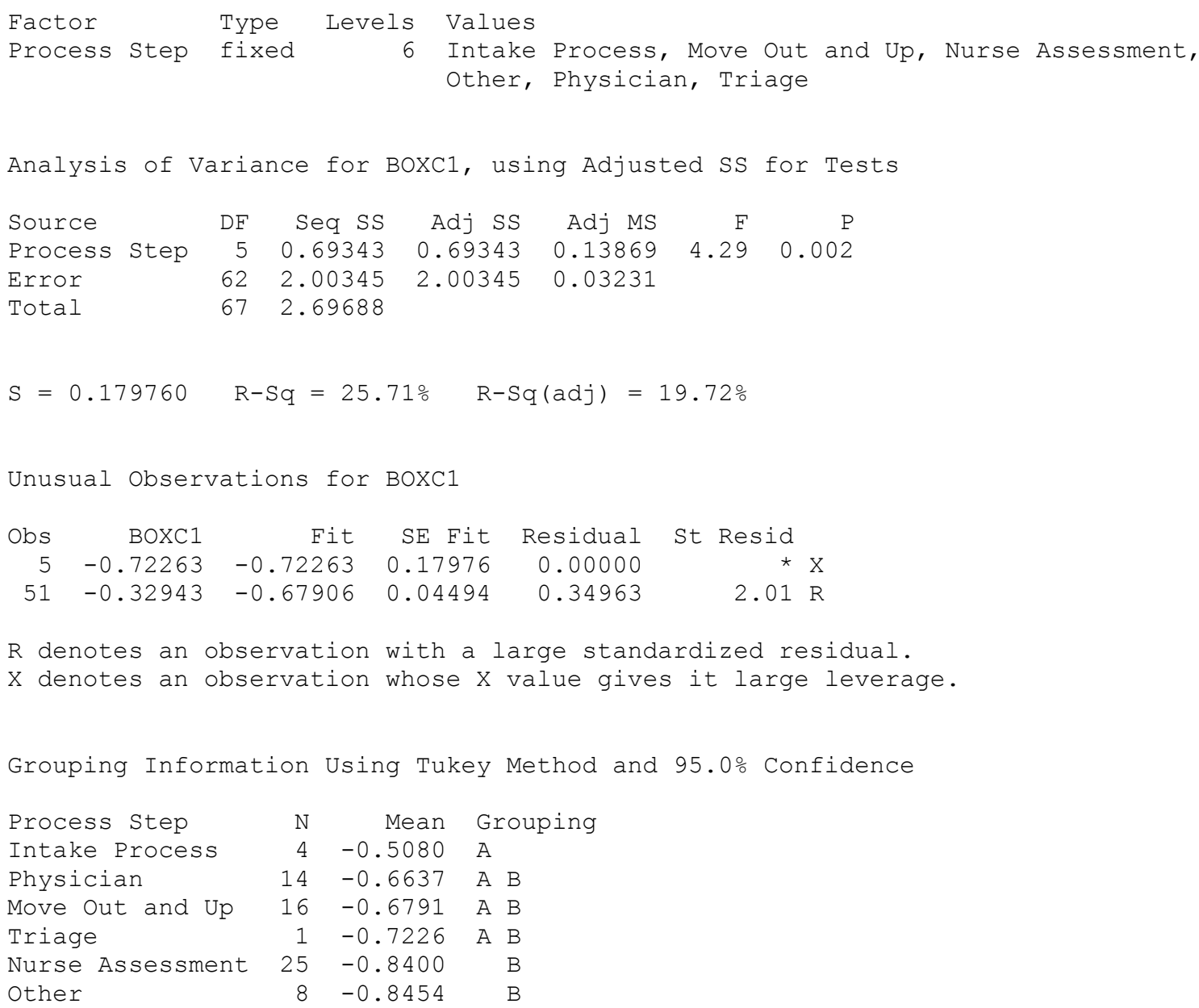

Figure 12: Analysis of Variance of the Macro Level Process Steps 


\section{APPENDIX C: INTERVIEW SCRIPT FOR NURSE MANAGERS, NURSES, AND UNIT SECRETARY}

Items:

- UofL ID

- Interview Script (x2)

- Notebook

- Information Sheet \& General Information Flyer

- SEIPS Model

- Process Flow Board w/Magnets

- Recorder w/Microphone

- Business Cards

\section{Opening script (START RECORDER):}

- Thank you for your willingness to participate

- Purpose: Gain a better understanding of how the AEU functions as a unit from your point-of-view.

- Specifically: We are interested in learning about the process flow starting from the time a primary care physician calls in to inform the AEU they are sending a patient until the patient leaves to go upstairs.

- With particular interest in the variations of the process that occur from patient to patient

- Duration: 60 minutes and will be audio-taped. In addition, I will also be making some note during...

- Information will be kept confidential and reported anonymously. Audio tape records will be destroyed after transcription.

- Please refrain from using patient, colleague, or clinics names during the interview.

- Prior Knowledge: I'd like you to try to explain things to me as if I didn't have this prior knowledge about patient care or the process.

- Your rights: You may decline to answer any of the questions and we can stop and restart the interview if the need arises. 


\section{- Do you have any questions before we get started?}

- System model/framework: First, I would like to start out by showing you the system model / framework that we are using.....the overall system is broken in to 5 primary components of...

QUESTION 1: Will you please tell me how long you have been a nurse.

P1: How long have you been a nurse at Kosair?

P2: How long have you worked in the AEU?

QUESTION 2: In your own words, please tell me what the purpose of the AEU is.

P1: Over time, has the purpose of the AEU changed? How has it changed?

QUESTION 3: Is there a written protocol for the AEU?

P1: Who can we get that written protocol from? OR Who is in control of the protocol?

P2: How was it determined what tasks make up the current protocols as it exists today? (ADMIN QUESTION)

P3: What are some common variations (workarounds) to the protocol that you experience?

P4: As you know, we have observed the AEU's many protocols; will you please tell us how you learned the protocols that you utilize in the AEU?

QUESTION 4: How are you evaluated in the AEU?

P1: Are there particular metrics that the AEU is measured on?

QUESTION 5: Using this whiteboard, I would like you to create the AEU process based on the steps you go through to process a patient based on your experiences of what happens on a daily basis.

P1: Please put in where you consistently see delays happen that effect the process $\checkmark$ Type of patient

seen in AEU?

Does written differ from what is done?

Do they know where to see the protocol themselves

Benchmarks used to evaluate AEU?

Written Metrics or just known 
P2: Based on the flow you just created, add the supporting department or personnel associated with each task.

P3: Will you please show me where the most prominent variation that affects the process is.

P4: Will you please clarify the process of the primary doctor calling Kosair to inform them that they are sending a patient to the AEU.

- When the primary doctor calls the AEU to inform them they are sending a patient, what type of information do they provide.

P5: Once the patient has arrived to the AEU, what percentage of the time is the diagnosis changed from that provide by the primary doctor.

- Will you please provide an example of this?

P6: How often are you provided with direct orders from the PCP calling in?

P7: Looking at the flow and personnel that you created on the whiteboard, who reports to whom. You can draw arrows between the personnel.

- So is ultimately in charge of the AEU, can this person tell someone who is performing a task in the AEU that this does not need to be performed there.

QUESTION 6: We have defined the process and talked about the minor variations within the process, now we are interested in learning about the inefficient processes. Will you please provide some examples of inefficient processes in the AEU?

P1: With the examples that you provided along with other processes we have seen in our observations and others provided by staff members, please rank the processes from most inefficient to least inefficient.

QUESTION 7: Will you please provide an instance where more than one doctor or nurse would enter a patient's room at different times and perform the same procedure or ask the same questions.

Could all patients have standing orders from their PCP

Who ultimately is in charge of the floor

$\square$ Identify 2 or 3 in charge and ask: Are they in charge based on function of their position of personality

$\square$ Possible significance test performed from this (Kruskal-

Wallis)

\section{$\square$ If wait on}

doctor is ranked high, ask: "do you think a full time doctor in the AEU is justified" 
P1: For a situation where this would occur, does it matter who enters the room first?

P2: What is the second set of information used for?

QUESTION 8: Will you please provide an instance where a doctor would NOT come to the AEU to see a patient.

QUESTION 9: If you could change one thing about the AEU, what would you change and why?

P1: Do you feel optimistic, pessimistic, or neutral that the change you would make could happen. Why? 
Wrap-up script: (Check all responses)

- That completes our questions, is there anything else that you haven't told me that you think is important for me to know?

- Thank you for participating in this interview. The information you have provided is very valuable to better understand the AEU.

- One last thing: after the interview is transcribe, would you like it to be emailed to you so that you may have an opportunity to elaborate on any point(s) that you feel are not clear?

- Findings / Outcomes from this study will be shared with your clinic

- Here is my contact information.

- Feel free to contact me if you think of anything else that may be relevant to the study or if you see me in the hospital, feel free to stop me.

Thank you again for your time. 


\section{APPENDIX D: INTERVIEW SCRIPT FOR DOCTOR INTERVIEW}

\section{Items:}

- UofL ID

- Interview Script (x2)

- Notebook

- Information Sheet \& General Information Flyer

- SEIPS Model

- Process Flow Board w/Magnets

- Recorder w/Microphone

- Business Cards

\section{Opening script (START RECORDER):}

- Thank you for your willingness to participate

- Purpose: Gain a better understanding of how the AEU functions as a unit from your point-of-view.

- Specifically: We are interested in learning about the process flow starting from the time a primary care physician calls in to inform the AEU they are sending a patient until the patient leaves to go upstairs.

- With particular interest in the variations of the process that occur from patient to patient

- Duration: 60 minutes and will be audio-taped. In addition, I will also be making some note during...

- Information will be kept confidential and reported anonymously. Audio tape records will be destroyed after transcription.

- Please refrain from using patient, colleague, or clinics names during the interview.

- Prior Knowledge: I'd like you to try to explain things to me as if I didn't have this prior knowledge about patient care or the process.

- Your rights: You may decline to answer any of the questions and we can stop and restart the interview if the need arises. 


\section{- Do you have any questions before we get started?}

- System model/framework: First, I would like to start out by showing you the system model / framework that we are using.....the overall system is broken in to 5 primary components of...

QUESTION 1: Will you please tell me how long you have been a doctor.

P1: How long have you been a doctor at Kosair?

P2: Concerning the AEU, how long have you been seeing patients in the AEU?

QUESTION 2: In your own words, please tell me what the purpose of the AEU is.

P1: Please tell us how you learned the protocols that you utilize in the AEU?

P2: Is there a written protocol for the AEU?

P3: Who can we get that written protocol from? OR Who is in control of the protocol?

P4: Over time, has the purpose of the AEU changed? How has it changed?

P5: What are some common variations (workarounds) to the protocol that you experience?

QUESTION 3: How are you evaluated in the AEU?

P1: Are there particular metrics that the AEU is measured on?

QUESTION 4: Using this whiteboard, I would like you to create the AEU process based on the steps you go through to process a patient based on your experiences of what happens on a daily basis.

P1: Based on the flow you just created, add the supporting department or personnel associated with each task.

P2: Please put in where you consistently see delays happen that effect the process
Type of patient seen in AEU?

Does written differ from what is done?

Do they know where to see the protocol themselves

Benchmarks used to evaluate AEU?

Written Metrics or just known

Could all patients have standing orders from 
P3: Will you please show me where the most prominent variation that affects the process is.

P4: From your point of view, will you please clarify the process of the primary doctor calling Kosair to inform them that they are sending a patient to the AEU.

- When the primary doctor calls the AEU to inform them they are sending a patient, what type of information do they provide.

P5: Once the patient has arrived to the AEU, what percentage of the time is the diagnosis changed from that provided by the primary doctor.

- Will you please provide an example of this?

P6: How often are you provided with direct orders from the PCP calling in?

P7: Looking at the flow and personnel that you created on the whiteboard, who reports to whom. You can draw arrows between the personnel.

- So is ultimately in charge of the AEU, can this person tell someone who is performing a task in the AEU that this does not need to be performed there.

QUESTION 5: We have defined the process and talked about the minor variations within the process, now we are interested in learning about the inefficient processes. Will you please provide some examples of inefficient processes in the AEU?

P1: With the examples that you provided along with other processes we have seen in our observations and others provided by staff members, please rank the processes from most inefficient to least inefficient.

QUESTION 6: Will you please provide an instance where more than one doctor or nurse would enter a patient's room at different times and perform the same procedure or ask the same questions.

P1: For a situation where this would occur, does it matter who their PCP

Who

ultimately is

in charge of

the floor

Identify 2 or 3

in charge and

ask: Are they

in charge

based on

function of

their position

of personality

Possible

significance

test performed

from this

(Kruskal-

Wallis) 
enters the room first?

P2: What is the second set of information used for?

QUESTION 7: Will you please provide an instance where a doctor would NOT come to the AEU to see a patient.

QUESTION 8: If you could change one thing about the AEU, what would you change and why?

P1: Do you feel optimistic, pessimistic, or neutral that the change you would make could happen. Why? 
Wrap-up script: (Check all responses)

- That completes our questions, is there anything else that you haven't told me that you think is important for me to know?

- Thank you for participating in this interview. The information you have provided is very valuable to better understand the AEU.

- One last thing: after the interview is transcribe, would you like it to be emailed to you so that you may have an opportunity to elaborate on any point(s) that you feel are not clear?

- Findings / Outcomes from this study will be shared with your clinic

- Here is my contact information.

- Feel free to contact me if you think of anything else that may be relevant to the study or if you see me in the hospital, feel free to stop me.

- Thank you again for your time. 


\section{APPENDIX E: INTERVIEW SCRIPT FOR ADMINISTRATOR INTERVIEW}

\section{Items:}

- UofL ID

- Interview Script (x2)

- Notebook

- Information Sheet \& General Information Flyer

- SEIPS Model

- Process Flow Board w/Magnets

- Recorder w/Microphone

- Business Cards

\section{Opening script (START RECORDER):}

- $\quad$ Thank you for your willingness to participate

- Purpose: Gain a better understanding of how the AEU functions as a unit from your point-of-view.

- Specifically: We are interested in learning about the process flow starting from the time a primary care physician calls in to inform the AEU they are sending a patient until the patient leaves to go upstairs.

- With particular interest in the variations of the process that occur from patient to patient

- Duration: 60 minutes and will be audio-taped. In addition, I will also be making some note during...

- Information will be kept confidential and reported anonymously. Audio tape records will be destroyed after transcription.

- $\quad$ Please refrain from using patient, colleague, or clinics names during the interview.

- Prior Knowledge: I'd like you to try to explain things to me as if I didn't have this prior knowledge about patient care or the process.

- Your rights: You may decline to answer any of the questions and we can stop and restart the interview if the need arises.

- Do you have any questions before we get started? 
- $\quad$ System model/framework: First, I would like to start out by showing you the system model / framework that we are using.....the overall system is broken in to 5 primary components of...

QUESTION 1: Will you please tell me how long you have been a doctor.

P1: How long have you been a doctor at Kosair?

P2: Concerning the AEU, have you ever seen patients in the AEU?

P3: Could you please describe your current position and how that relates to the AEU.

P4: Based on your current relationship with the AEU, would you describe your role as being a reviewer or an evaluator of the area and/or personnel.

QUESTION 2: In your own words, please tell me what the purpose of the AEU is.

P1: Please tell us how you learned the protocols utilized in the AEU?

P2: Is there a written protocol for the AEU?

P3: Who can we get that written protocol from? OR Who is in control of the protocol?

P4: Over time, has the purpose of the AEU changed? How has it changed?

P5: What are some common variations (workarounds) to the protocol that you are aware of?

QUESTION 3: How is the AEU evaluated?

P1: Are there particular metrics that the AEU is measured on?

QUESTION 4: Using this whiteboard, I would like you to create the AEU process based on your understanding of what happens on a daily basis.

P1: Based on the flow you just created, add the supporting department or personnel associated with each task.

How many positions?

Type of patient seen in $A E U$ ?

Does written differ from what is done?

Do they know where to see the protocol themselves

Benchmarks used to evaluate AEU?

Written Metrics or just known

Who ultimately is in charge of the floor

Identify 2 or 3 in charge 
P2: Please put in where delays are known to consistently occur.

P3: Will you please show me where the most prominently known variations occur that affect the process.

P4: Based on your understanding of the AEU process, will you please clarify the process of the primary doctor calling Kosair to inform them that they are sending a patient to the AEU.

- When the primary doctor calls the AEU to inform them they are sending a patient, what type of information do they provide.

- How does the primary doctor know what process to follow.

P5: Looking at the flow and personnel that you created on the whiteboard, who reports to whom. You can draw arrows between the personnel.

- So is ultimately in charge of the

AEU, can this person tell someone who is performing a task in the AEU that this does not need to be performed there.

QUESTION 5: We have defined the process and talked about the minor variations within the process, now we are interested in learning about the inefficient processes. From an external standpoint, will you please provide some examples of inefficient processes in the AEU?

P1: With the examples that you provided along with other processes we have seen in our observations and others provided by staff members, please rank the processes from most inefficient to least inefficient.

QUESTION 6: Will you please provide an instance where more than one doctor or nurse would enter a patient's room at different times and perform the same procedure or ask the same questions.

P1: For a situation where this would occur, does it matter who enters the room first?

P2: What is the second set of information used for?

QUESTION 7: Will you please provide an instance where a doctor 
would NOT come to the AEU to see a patient.

QUESTION 8: If you could change one thing about the AEU, what would you change and why?

P1: Do you feel optimistic, pessimistic, or neutral that the change you would make could happen. Why? 
Wrap-up script: (Check all responses)

- That completes our questions, is there anything else that you haven't told me that you think is important for me to know?

- Thank you for participating in this interview. The information you have provided is very valuable to better understand the AEU.

- One last thing: after the interview is transcribe, would you like it to be emailed to you so that you may have an opportunity to elaborate on any point(s) that you feel are not clear?

- Findings / Outcomes from this study will be shared with your clinic

- Here is my contact information.

- Feel free to contact me if you think of anything else that may be relevant to the study or if you see me in the hospital, feel free to stop me.

- Thank you again for your time. 


\title{
CURRICULUM VITAE
}

\section{SCOTT R. HOOVER}

\author{
9103 Crowne Springs Circle Apt. 301 \\ Louisville, KY, 40241 \\ Tel: (270) 792-2009 (Cell) \\ Email: scott.r.hoover@gmail.com
}

\section{EDUCATION}

RESEARCH INTERESTS

PUBLICATIONS

\author{
University of Louisville, Louisville, KY, \\ PhD Candidate in Industrial Engineering, Expected Graduation: Summer 2013 \\ Specialization: Prospective Risk Assessment \\ Advisor: Dr. Grady T. Holman \& Dr. Gail W. DePuy \\ Dissertation: 'Evaluation of a Children's Hospital's Direct Admission Process to Improve \\ Outcomes and Efficiency of Care' \\ M.S. in Industrial Engineering, 2010 \\ Specialization: Computer Simulation \\ Advisor: Dr. Gail W. DePuy \\ Thesis: 'Simulation Analysis of Resident and Attending Physician Teaching Methods at \\ Kosair Children's Hospital' \\ B.A. in Mathematics, 2009
}

1. Analysis, quantification, and creation of computer simulation models of healthcare processes and procedures for the purpose of improving methods and procedures.

Current Research: Development of a "real world" primary care clinic model based on multiple physician-nurse teams treating patients simultaneously.

2. Performing prospective risk assessment of healthcare processes for continuous improvement of patient safety.

Current Research: Evaluation of a children's hospital's rapid direct admissions unit.

\section{Manuscripts in Review:}

1. Hoover, S., Wooldridge, A., Wetterneck, T., Holman, G.T., Need for Alternative Simulation Methods for Modeling a Primary Care Physician. IIE Transactions on Healthcare Systems

2. Holman, G.T., Hoover, S., and Maghsoodloo, S., Selling the Word "Ergonomics" or the Benefit of, which Changes Buyer Intent? International Journal of Industrial Ergonomics

\section{Manuscripts in Draft:}

1. Wooldridge, A., Hoover, S., Wetterneck, T., Holman, G.T., Development of a Primary Care Nurse Task List to Evaluate Clinic Workflow.

2. Wooldridge, A., Hoover, S., Wetterneck, T., Holman, G.T., Development of a Primary Care Nurse Task-Based Simulation Model to Analyze Workflow.

3. Gentry, E., Hoover, S., A Day in the Emergency Department: How Perception is Different than Reality.

4. Hoover, S., Gentry, E., Evaluation of Teaching Methods within an Emergency Department: A Case Study 
PRESENTATIONS

GRANTS

ACADEMIC EXPERIENCE

Conference Presentations and Posters:

1. Wooldridge, A., Hoover, S. Holman, G.T., 2013, Evaluation of Current Simulation Methods in Healthcare and their Impact, Proceedings of the 2013 Healthcare Systems Process Improvement Conference, New Orleans, LA, poster.

\section{Invited Presentations:}

1. Hoover, S., Wooldridge, A., Holman, G.T., 2012, Simulating Physician-Nurse Teams in a Primary Care Clinic at the Task Level, University of Wisconsin - Madison Department of Industrial and Systems Engineering, Madison, WI.

2. Hoover, S., 2011, Simulation Analysis of Teaching Methods at Kosair Children's Hospital, The American Society for Quality Monthly Meeting, Louisville, KY.

3. Hoover, S., 2011, Simulation Analysis of Resident and Attending Physician Teaching Methods at Kosair Children's Hospital, Kosair Children's Hospital Directors Meeting, Louisville, KY.

\section{Recently Submitted Proposals}

1. Holman, G.T. (PI), 2012, "Analysis and Redesign of a Hospital's Admissions Process", Intramural Research Incentive Grants, University of Louisville, $\$ 10,000$.

2. Holman, G.T. (PI), 2011, "Developing Evidence-Based Simulation for Evaluating EHR \& Non-EHR Clinic Workflow", NIOSH Deep South Center for Occupational Safety and Health: Education \& Research Center, $\$ 11,880$.

Instructor, Department of Industrial Engineering, University of Louisville, Louisville, KY Scheduled Summer 2013.

IE 541 - Simulation: Prepared material, lectured, and evaluated students based on the use of computer simulation to analyze systems. Topics covered include Monte Carlo techniques, sampling from and identifying stochastic distributions, estimating performance measures form simulation outputs, validation methods, and ARENA simulation language.

Graduate Teaching Assistant, Department of Industrial Engineering, University of Louisville, Louisville, KY, 2009-present

Developed course material and provided guest lectures, grading, and other tasks:

- Techniques in the Workplace (undergraduate, graduate), 2010, 2012, 2013

- Engineering Economics Analysis (undergraduate), 2012

- Principles of Occupational Ergonomics (undergraduate, graduate), 2010-2012

- Experimental Design in Engineering (undergraduate, graduate), 2011

Course where primary duties include grading of homework, labs, and exams:

- Probability and Statistics for Engineers (undergraduate), 2009

- Operations Research Methods(undergraduate, graduate), 2009, 2010

- Management of Human Systems (graduate), 2010

- Production and Inventory Control (undergraduate), 2010, 2011

- Fundamentals of Industrial Engineering (undergraduate), 2011, 2012

- Quality Control (undergraduate), 2012

- Work Design (undergraduate), 2012

- Models of Logistic Systems (graduate), 2013

- Lean Systems (undergraduate, graduate), 2013 
Laboratory Technician, Center for Ergonomics, University of Louisville, 2010-present Assisted in building and maintaining Industrial Engineering's ergonomics and human factors laboratory. Laboratory capabilities include simultaneous capture of ground force reaction, motion capture, and balance.

Equipment: TrakStar (Magnetic Motion Capture System)

$$
\begin{aligned}
& \text { Bertec Forceplates } \\
& \text { SR EyeTrack }
\end{aligned}
$$

INDUSTRIAL

EXPERIENCE

SERVICE

PROFESSIONAL
Industrial \& Systems Engineer, Advanced Solutions, Louisville, KY, Feb 2012-present Lead the simulation development team in the completion of simulation projects. Projects include:

- General Electric Appliance Park, Feb 2012. Created computer simulation model of new washing machine assembly line to determine possible bottlenecks within the system.

- CEO of Advanced Solutions, May 2012. At the CEO's request, developed computer simulation model for the Sales team of Advanced Solutions for purposes of advertising computer simulation modeling.

- Herbalife International, Oct 2012. Created computer simulation model of production line to assess the effects of new machinery and additional production lines.

\section{To the Profession}

Organizations

- Graduate Student Council, University of Louisville, Louisville, KY, 2011-present Department of Industrial Engineering Representative

To the University of Louisville

Recruitment

- INSPIRE Program (2011-2012)

- Minorities to Engineering

- Speed School Career Day (2010-2012)

- Speed School perspective student tours (2011-2012)

\section{Memberships}

1. Institute of Industrial Engineers, 2009-Present

2. Human Factors and Ergonomics Society, 2010-Present

3. Alpha Pi Mu Honor Society, 2011-Present

- Chapter President, 2012-Present

\section{Awards}

1. The American Society for Quality Award, 2011

2. Presidential Scholarship, 2005-2009 